\title{
Modified Suliciu relaxation system and exact resolution of isolated shock waves
}

\author{
Christophe Chalons \\ Université Paris Diderot-Paris 7 \& Laboratoire J.-L. Lions, U.M.R. 7598 \\ UMPC, Boîte courrier 187, 75252 Paris Cedex 05, France. \\ E-mail: christophe chalons@ljll.univ-paris-diderot.fr \\ Frédéric Coquel \\ CNRS $\&$ Centre de Mathématiques Appliquées, U.M.R. 7641 \\ Ecole Polytechnique, Route de Saclay, 91128 Palaiseau Cedex, France. \\ E-mail: frederic.coquel@cmap.polytechnique.fr
}

\section{Abstract}

We present a new Approximate Riemann Solver (ARS) for the gas dynamics equations in Lagrangian coordinates and with general non linear pressure laws. The design of this new ARS relies on a generalized Suliciu pressure relaxation approach. It gives by construction the exact solutions for isolated entropic shocks and we prove that it is Lipschitzcontinuous and satisfies an entropy inequality. Finally, the ARS is used to develop either a classical entropy conservative Godunov-type method, or a Glimm-type (random sampling based Godunov-type) method able to generate infinitely sharp discrete shock profiles. Numerical experiments are proposed to prove the validity of these approaches.

\section{Introduction}

In this paper we consider the following non linear system of barotropic gas dynamics equations in Lagrangian coordinates :

$$
\left\{\begin{array}{l}
\partial_{t} \tau-\partial_{x} u=0, \\
\partial_{t} u+\partial_{x} p(\tau)=0,
\end{array} \quad(x, t) \in \mathbb{R} \times \mathbb{R}^{+*},\right.
$$

where $\tau>0$ represents the inverse of a density, $u$ is the velocity and $p=p(\tau)>0$ is the pressure of the fluid. We assume that $p^{\prime}(\tau)$ is negative for all $\tau>0$ so that the system is known to be strictly hyperbolic with characteristic speeds $\lambda^{(-1)}=\lambda^{(-1)}(\tau)$ and $\lambda^{(1)}=\lambda^{(1)}(\tau)$ given by

$$
\lambda^{(-1)}(\tau)=-\sqrt{-p^{\prime}(\tau)}, \quad \lambda^{(1)}(\tau)=\sqrt{-p^{\prime}(\tau)}
$$

over the phase space $\Omega$ given by

$$
\Omega=\left\{\mathbf{v}=(\tau, u)^{t} \in \mathbb{R}^{2}, \tau>0, u \in \mathbb{R}\right\} .
$$


We introduce the following short form for (11) supplemented with a given initial condition $\mathbf{v}_{0}$ at time $t=0$ :

$$
\left\{\begin{array}{l}
\partial_{t} \mathbf{v}+\partial_{x} \mathbf{g}(\mathbf{v})=0, \\
\mathbf{v}(x, 0)=\mathbf{v}_{0}(x),
\end{array} \quad(x, t) \in \mathbb{R} \times \mathbb{R}^{+*}, \mathbf{v}(x, t) \in \Omega\right.
$$

It is now very well-known that (3) generally does not admit smooth solutions for large times so that weak solutions in the sense of distributions have to be considered. Due to the presence of discontinuities, these are generally not uniquely determined by $\mathbf{v}_{0}$ and the validity of an entropy criterion is added for the admissibility of discontinuities. More precisely, (3) is supplemented with the following entropy inequality to be satisfied in the sense of distributions :

$$
\partial_{t} \mathcal{U}(\mathbf{v})+\partial_{x} \mathcal{F}(\mathbf{v}) \leq 0
$$

where $(\mathcal{U}, \mathcal{F})$ is the entropy-entropy flux pair given by

$$
\mathcal{U}(\mathbf{v})=\frac{u^{2}}{2}+e(\tau), \quad \mathcal{F}(\mathbf{v})=p u
$$

with

$$
e(\tau)=-\int^{\tau} p(y) d y
$$

Note that $\mathcal{U}(\mathbf{v})$ is strictly convex by assumption on the derivative of $p(\tau)$. Throughout this paper, we also assume that the pressure $p$ is a twice continuously differentiable and strictly convex function, that is $p^{\prime \prime}(\tau)>0$ for all $\tau>0$, so that the characteristic fields associated with $\lambda^{(-1)}$ and $\lambda^{(1)}$ are genuinely nonlinear (GNL) (see for instance 14]). From a theoretical point of view, existence and uniqueness of an entropy solution is then proved for the Cauchy problem (3)-(4). We refer for instance the reader to [3] for a review. In particular, if we consider a Riemann problem connecting two states $\mathbf{v}_{L}$ and $\mathbf{v}_{R}$ in $\Omega$ and associated with the following initial data

$$
\mathbf{v}_{0}(x)=\left\{\begin{array}{lll}
\mathbf{v}_{L} & \text { for } \quad x<0 \\
\mathbf{v}_{R} & \text { for } \quad x>0
\end{array}\right.
$$

the solutions $(x, t) \rightarrow \mathbf{v}\left(x / t ; \mathbf{v}_{L}, \mathbf{v}_{R}\right)$ have the self-similar structure shown in Figure 1 where the dotted lines stand for the so-called simple waves associated with $\lambda^{(-1)}$ and $\lambda^{(1)}$. In other words, the initial state $\mathbf{v}_{L}$ (respectively $\mathbf{v}_{R}$ ) is connected to the right (resp. left) to an intermediate state $\mathbf{v}_{*}$ by either an entropy shock wave or a smooth rarefaction wave.

From a numerical point of view, the celebrated Godunov method is an example of conservative and entropy satisfying numerical strategy that provides good approximations of the entropy solution to (3)-(4). Recall indeed that the Lax-Wendroff theorem (see [14] for details) ensures a convergence to the unique entropy solution provided that the method does converge. For the sake of completeness and to fix the notations, we will review the procedure in details in Section 2 below, but let us briefly recall that the method is made of two steps 


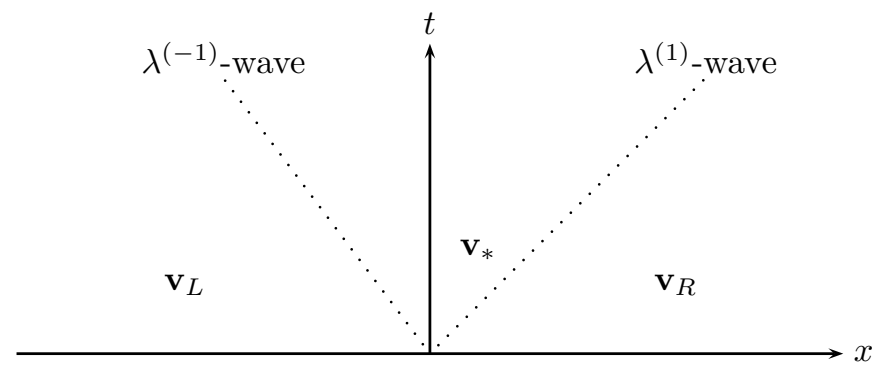

Figure 1: Typical wave structure of a Riemann solution of (3)-(4).

to make evolve piecewise constant approximate solutions from a time $t_{1}$ to a next time $t_{2}$ : a first step in which the solution at time $t_{1}$ evolves from time $t_{1}$ to time $t_{2}$ according to the PDE model under consideration, namely (3)-(4), and a second step of projection onto piecewise constant functions. In the first step and provided that $t_{1}$ and $t_{2}$ are sufficiently close, the solution to (3)-(4) is made of the juxtaposition of Riemann problem solutions set at each point of discontinuity of the solution at time $t_{1}$. In the classical Godunov method the solutions of these Riemann problems (the self-similar structure of which being given on Figure 1) are exactly calculated, which may be expensive for general non linear pressure laws $p=p(\tau)$.

In order to design cheaper Godunov-type methods, Approximate Riemann Solvers are often introduced and used in the first step of the method. The exact simple waves are then approximated in such a way that the underlying approximate Riemann solutions are easily calculable and consistent with the exact solution in the integral sense of Harten-Lax-Van Leer 13. Among the well-known ARS, and without any attempt to be exhaustive, are the HartenLax-Van Leer's ARS [13, the Roe's ARS [18] and the ARS based on a Suliculike pressure relaxation procedure as the ones proposed in [19] (see also [15], [10, 12, 4], 1], 2], 7]...). Two will be briefly reviewed in the next sections. Note right now that they generally give rise to accurate and entropy numerical solutions but they are not able to provide exact Riemann solutions, even in the simple case of an isolated shock wave (the Roe's ARS does it but is not naturally entropic without correction). More precisely, if the Riemann solution $\mathbf{v}(x, t)$ to (3)-(4) and initial data (6) simply consists of an isolated shock wave between $\mathbf{v}_{L}$ and $\mathbf{v}_{R}$ propagating with velocity $s$, that is

$$
\mathbf{v}(x, t)= \begin{cases}\mathbf{v}_{L} & \text { for } \quad x<s t, \\ \mathbf{v}_{R} & \text { for } \quad x>s t,\end{cases}
$$

then the Riemann solution $(x, t) \rightarrow \tilde{\mathbf{v}}\left(x / t ; \mathbf{v}_{L}, \mathbf{v}_{R}\right)$ given by the ARS under consideration is actually an approximation in the sense that $\mathbf{v}\left(. ; \mathbf{v}_{L}, \mathbf{v}_{R}\right)$ and $\tilde{\mathbf{v}}\left(. ; \mathbf{v}_{L}, \mathbf{v}_{R}\right)$ do not coincide. In this paper, our objective is to propose a new class of ARS that is able to exactly calculate such simple solutions. Our strat- 
egy is based on a suitable generalization of the Sulicu-like pressure relaxation approache and is shown to be entropy satisfying. We refer the reader to [2] for similar results at the semi-discrete level and using different strategies.

The outline of the paper is as follows. We first briefly review the celebrated Godunov method and two approximate Godunov-type methods. Section 3 presents a new Approximate Riemann Solver based on a generalized Suliciu pressure relaxation approach. At last, Section 4 gives the main properties satisfied by this new ARS and Section [5 is devoted to numerical experiments.

\section{Exact and approximate Godunov-type meth- ods}

For the sake of completeness and in order to fix the notations, we briefly review in this section the exact or approximate Godunov-type methods in details. We consider here a given set of $l \times l$ hyperbolic equations, $l>1$, that we write in the following condensed form

$$
\partial_{t} \mathbf{u}+\partial_{x} \mathbf{f}(\mathbf{u})=0
$$

supplemented with the validity of an entropy inequality

$$
\partial_{t} \eta(\mathbf{u})+\partial_{x} q(\mathbf{u}) \leq 0
$$

where $(\eta, q)$ is a strictly convex entropy-entropy flux pair. The eigenvalues are noted $\left(\lambda^{(k)}(\mathbf{u})\right)_{k}$. Let us supplement (7)-(8) with an initial data $\mathbf{u}_{0}$.

Let us introduce a space step $\Delta x$ and a time step $\Delta t$, bothassumed to be constant for simplicity. We set $\nu=\Delta t / \Delta x$. Then, we define the mesh interfaces $x_{j+1 / 2}=j \Delta x$ for $j \in \mathbb{Z}$ and the intermediate times $t^{n}=n \Delta t$ for $n \in \mathbb{N}$, and we seek at each time $t^{n}$ an approximation $\mathbf{u}_{j}^{n}$ of the solution of (7)-(8) on the interval $\left[x_{j-1 / 2}, x_{j+1 / 2}\right), j \in \mathbb{Z}$. Therefore, a piecewise constant approximate solution $x \rightarrow \mathbf{u}_{\nu}\left(x, t^{n}\right)$ of the solution $\mathbf{u}$ is given by

$$
\mathbf{u}_{\nu}\left(x, t^{n}\right)=\mathbf{u}_{j}^{n} \text { for all } x \in C_{j}=\left[x_{j-1 / 2}, x_{j+1 / 2}\right), \quad j \in \mathbb{Z}, n \in \mathbb{N} .
$$

When $n=0$, we set $x_{j}=\left(x_{j-1 / 2}+x_{j+1 / 2}\right) / 2$ and

$$
\mathbf{u}_{j}^{0}=\frac{1}{\Delta x} \int_{x_{j-1 / 2}}^{x_{j+1 / 2}} \mathbf{u}_{0}(x) d x, \text { for all } j \in \mathbb{Z} .
$$

Assuming as given a sequence $\left(\mathbf{u}_{j}^{n}\right)_{j \in \mathbb{Z}}$ at time $t^{n}$, it is now a question of proposing a way of advancing it to the next time level $t^{n+1}$.

\subsection{Exact Godunov method}

We first use the celebrated Godunov scheme in its classical form. As briefly recalled in the introduction, the Godunov scheme is composed of two steps : 
a first step in which initial data evolves in time according to the PDE model under consideration, and a second step of projection onto piecewise constant functions.

Step 1: Evolution in time

In this first step, one solves the following Cauchy problem

$$
\left\{\begin{array}{l}
\partial_{t} \mathbf{u}+\partial_{x} \mathbf{f}(\mathbf{u})=0, \quad x \in \mathbb{R} \\
\mathbf{u}(x, 0)=\mathbf{u}_{\nu}\left(x, t^{n}\right)
\end{array}\right.
$$

for times $t \in[0, \Delta t]$. Recall that $x \rightarrow \mathbf{u}_{\nu}\left(x, t^{n}\right)$ is piecewise constant. Then, under the usual CFL restriction

$$
\frac{\Delta t}{\Delta x} \max _{k, \mathbf{u}}\left\{\left|\lambda^{(k)}(\mathbf{u})\right|\right\} \leq \frac{1}{2},
$$

for all the $\mathbf{u}$ under consideration, the solution of (9) is known by gluing together the solutions of the Riemann problems set at each interface. More precisely

$$
\mathbf{u}(x, t)=\mathbf{u}\left(\frac{x-x_{j+1 / 2}}{t} ; \mathbf{u}_{j}^{n}, \mathbf{u}_{j+1}^{n}\right) \text { for all }(x, t) \in\left[x_{j}, x_{j+1}\right] \times[0, \Delta t],
$$

where $(x, t) \rightarrow \mathbf{u}\left(x / t ; \mathbf{u}_{L}, \mathbf{u}_{R}\right)$ denotes the self-similar solution to the Riemann problem

$$
\left\{\begin{array}{l}
\partial_{t} \mathbf{u}+\partial_{x} \mathbf{f}(\mathbf{u})=0, \quad x \in \mathbb{R}, \quad t \in \mathbb{R}^{+, *} \\
\mathbf{u}(x, 0)=\left\{\begin{array}{lll}
\mathbf{u}_{L} & \text { if } & x<0, \\
\mathbf{u}_{R} & \text { if } & x>0,
\end{array}\right.
\end{array}\right.
$$

whatever $\mathbf{u}_{L}$ and $\mathbf{u}_{R}$ are in the phase space.

Step 2: Projection $\left(t^{n} \rightarrow t^{n+1}\right)$

Aim of this second step is to get a piecewise constant approximate solution on each cell $\mathcal{C}_{j}$ at time $t^{n+1}$. This may be simply done by averaging the solution $x \rightarrow \mathbf{u}(x, \Delta t)$ given by (11), as expressed by the following update formula :

$$
\mathbf{u}_{j}^{n+1}=\frac{1}{\Delta x} \int_{x_{j-1 / 2}}^{x_{j+1 / 2}} \mathbf{u}(x, \Delta t) d t, j \in \mathbb{Z} .
$$

Actually, it is well-known that one can provide an even simpler formula for $\mathbf{u}_{j}^{n+1}$ from Green's theorem and using (9) and (13). More precisely we also have

$$
\mathbf{u}_{j}^{n+1}=\mathbf{u}_{j}^{n}-\frac{\Delta t}{\Delta x}\left(\mathbf{f}_{j+1 / 2}^{n}-\mathbf{f}_{j-1 / 2}^{n}\right) \text { for all } j \in \mathbb{Z},
$$

where the numerical fluxes are given by

$$
\mathbf{f}_{j+1 / 2}^{n}=\mathbf{f}\left(\mathbf{u}\left(0^{ \pm} ; \mathbf{u}_{j}^{n}, \mathbf{u}_{j+1}^{n}\right)\right) \text { for all } j \in \mathbb{Z},
$$

and classical notations have been used for the traces at $0^{-}$and $0^{+}$of the Riemann solutions under consideration. Note in passing that we necessarily have for all $j \in \mathbb{Z}$ :

$$
\mathbf{f}\left(\mathbf{u}\left(0^{-} ; \mathbf{u}_{j}^{n}, \mathbf{u}_{j+1}^{n}\right)\right)=\mathbf{f}\left(\mathbf{u}\left(0^{+} ; \mathbf{u}_{j}^{n}, \mathbf{u}_{j+1}^{n}\right)\right) .
$$

That is indeed obvious if $\mathbf{u}\left(0^{-} ; \mathbf{u}_{j}^{n}, \mathbf{u}_{j+1}^{n}\right)=\mathbf{u}\left(0^{+} ; \mathbf{u}_{j}^{n}, \mathbf{u}_{j+1}^{n}\right)$ while in the opposite case, the associated discontinuity is necessarily stationary and (15) follows from Rankine-Hugoniot conditions. In other words, the method is conservative at the corresponding interface $j+1 / 2$. We refer again to [14 for more details. 


\subsection{Approximate Godunov-type methods}

The design principle of Godunov-type method is to replace the exact solution to the Riemann problems set at each interface by an approximate solution. We briefly recall in this section the notion of consistency in the integral sense of such a self-similar approximate Riemann solution using the same notations as above. We also derive the corresponding approximate Godunov-type schemes for approximating the solutions to (7) and refer for instance to [14] for the details.

Solving the Riemann problem amounts to find the solution to (12). Let us consider a simple approximate Riemann solver $(x, t) \rightarrow \tilde{\mathbf{u}}\left(x / t ; \mathbf{u}_{L}, \mathbf{u}_{R}\right)$ made of $l+1$ constant states $\mathbf{u}_{k}$ separated by discontinuities propagating with velocities $\lambda_{k}=\lambda_{k}\left(\mathbf{u}_{L}, \mathbf{u}_{R}\right)$, namely

$$
\tilde{\mathbf{u}}\left(\frac{x}{t} ; \mathbf{u}_{L}, \mathbf{u}_{R}\right)= \begin{cases}\mathbf{u}_{0}=\mathbf{u}_{L}, & \frac{x}{t}<\lambda_{1} \\ \vdots & \\ \mathbf{u}_{k}, & \lambda_{k}<\frac{x}{t}<\lambda_{k+1} \\ \vdots & \\ \mathbf{u}_{l}=\mathbf{u}_{R}, & \frac{x}{t}>\lambda_{l}\end{cases}
$$

If $\Delta x>0$ and $\Delta t>0$ are respectively the constant space and time steps that verify the CFL condition

$$
\max _{1 \leq k \leq l}\left|\lambda_{k}\left(\mathbf{u}_{L}, \mathbf{u}_{R}\right)\right| \frac{\Delta t}{\Delta x} \leq \frac{1}{2}
$$

the approximate Riemann solver is said to be consistent with the integral form of (17) over the interval $\left[-\frac{\Delta x}{2}, \frac{\Delta x}{2}\right]$ if the integral of (16) equals the integral of the exact solution which equivalently writes

$$
\mathbf{f}\left(\mathbf{u}_{R}\right)-\mathbf{f}\left(\mathbf{u}_{L}\right)=\sum_{k=1}^{l} \lambda_{k}\left(\mathbf{u}_{L}, \mathbf{u}_{R}\right)\left(\mathbf{u}_{k}-\mathbf{u}_{k-1}\right)
$$

Then, the approximate Godunov scheme reads

$$
\left\{\begin{array}{l}
\mathbf{u}_{j}^{n+1}=\mathbf{u}_{j}^{n}-\frac{\Delta t}{\Delta x}\left(\mathbf{f}_{j+\frac{1}{2}}^{n}-\mathbf{f}_{j-\frac{1}{2}}^{n}\right) \\
\mathbf{f}_{j+\frac{1}{2}}^{n}=\mathbf{f}\left(\mathbf{u}_{j}^{n}, \mathbf{u}_{j+1}^{n}\right)
\end{array}\right.
$$

with

$$
\mathbf{f}\left(\mathbf{u}_{L}, \mathbf{u}_{R}\right)=\frac{1}{2}\left\{\mathbf{f}\left(\mathbf{u}_{L}\right)+\mathbf{f}\left(\mathbf{u}_{R}\right)-\sum_{k=1}^{l}\left|\lambda_{k}\left(\mathbf{u}_{L}, \mathbf{u}_{R}\right)\right|\left(\mathbf{u}_{k}-\mathbf{u}_{k-1}\right)\right\} .
$$

As far as the consistency with the entropy inequality (8) is concerned, the simple approximate Riemann solver is said to be consistent with the integral form of 
(8) if and only if under the CFL condition (17) we have

$$
q\left(\mathbf{u}_{R}\right)-q\left(\mathbf{u}_{L}\right) \leq \sum_{k=1}^{l} \lambda_{k}\left(\mathbf{u}_{L}, \mathbf{u}_{R}\right)\left(\eta\left(\mathbf{u}_{k}\right)-\eta\left(\mathbf{u}_{k-1}\right)\right) .
$$

Then, the numerical scheme defined by (19) satisfies the following discrete entropy inequality

$$
\left\{\begin{array}{l}
\eta\left(\mathbf{u}_{j}^{n+1}\right) \leq \eta\left(\mathbf{u}_{j}^{n}\right)-\frac{\Delta t}{\Delta x}\left(q_{j+\frac{1}{2}}^{n}-q_{j-\frac{1}{2}}^{n}\right), \\
q_{j+\frac{1}{2}}^{n}=q\left(\mathbf{u}_{j}^{n}, \mathbf{u}_{j+1}^{n}\right)
\end{array}\right.
$$

with

$$
q\left(\mathbf{u}_{L}, \mathbf{u}_{R}\right)=\frac{1}{2}\left\{q\left(\mathbf{u}_{L}\right)+q\left(\mathbf{u}_{R}\right)-\sum_{k=1}^{l}\left|\lambda_{k}\left(\mathbf{u}_{L}, \mathbf{u}_{R}\right)\right|\left(\eta\left(\mathbf{u}_{k}\right)-\eta\left(\mathbf{u}_{k-1}\right)\right)\right\} .
$$

The CFL condition associated with this explicit in time Godunov-type scheme naturally reads

$$
\max _{1 \leq k \leq l}\left|\lambda_{k}\left(\mathbf{u}_{j}^{n}, \mathbf{u}_{j+1}^{n}\right)\right| \frac{\Delta t}{\Delta x} \leq \frac{1}{2},
$$

for all $j$. Again, we refer to [14] for more details.

\subsection{Examples of Approximate Riemann Solvers}

We briefly review in this section two celebrated approximate Riemann solvers, namely the Harten, Lax van Leer (HLL) 13 and the Suliciu relaxation 19. Riemann solvers. They are both known to generally give rise to accurate and entropy numerical solutions but as already said in the introduction, they are not able to provide exact Riemann solutions in the simple situation of an isolated shock wave. An extension of the Suliciu relaxation Riemann solver will be proposed in the next sections to overcome this shortcoming.

\subsubsection{HLL Approximate Riemann Solver}

The approximate Riemann solver proposed by Harten, Lax and van Leer in [13. corresponds to the choice $l=1$ :

$$
\tilde{\mathbf{u}}\left(\frac{x}{t} ; \mathbf{u}_{L}, \mathbf{u}_{R}\right)= \begin{cases}\mathbf{u}_{L}, & \frac{x}{t}<\lambda_{1} \\ \mathbf{u}_{*}, & \lambda_{1}<\frac{x}{t}<\lambda_{2} \\ \mathbf{u}_{R}, & \frac{x}{t}>\lambda_{2}\end{cases}
$$

where the consistency relation (18) easily yields

$$
\mathbf{u}_{*}=\frac{\lambda_{2} \mathbf{u}_{R}-\lambda_{1} \mathbf{u}_{L}}{\lambda_{2}-\lambda_{1}}-\frac{\mathbf{f}\left(\mathbf{u}_{R}\right)-\mathbf{f}\left(\mathbf{u}_{L}\right)}{\lambda_{2}-\lambda_{1}} .
$$


The typical structure is then as represented on Figure 2 below. This simple approximate Riemann solver is shown to be consistent with the integral form of (8) under the CFL condition (17) provided that $\lambda_{1}$ and $\lambda_{2}$ satisfy the so-called sub-characteristic condition

$$
\lambda_{1}<\lambda^{(k)}(\tau)<\lambda_{2}, \quad \text { for all } k=-1,1, \quad \text { for all } \tau,
$$

where $\lambda^{(k)}(\tau), k=-1,1$ are given by (2) and for all the values of $\tau$ involved in the exact Riemann solution. In other words, the information has to propagate faster in the approximate solution than in the exact one.

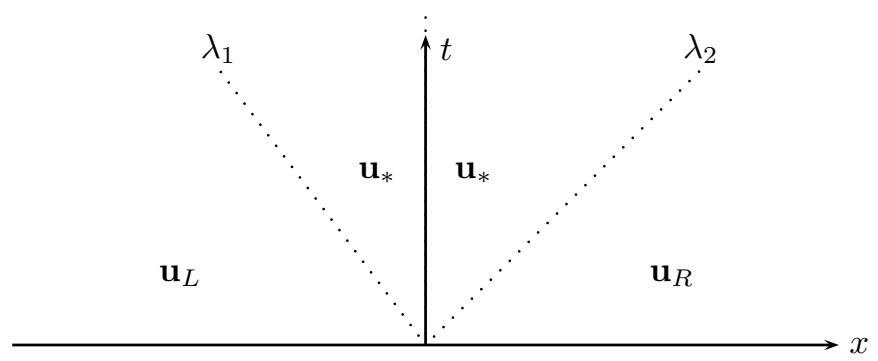

Figure 2: Typical wave structure of a HLL approximate Riemann solution to (12).

\subsubsection{Suliciu Relaxation ARS}

Approximate Riemann solvers based on the so-called relaxation approach are nothing but exact Riemann solvers but for a suitable extended system. By suitable, we mean in particular that the enlarged hyperbolic model will have the property of being non linear with only linearly degenerate fields, so that the Riemann solutions are uniquely made of discontinuities and can be explicitly calculated. Motivated by the work of Chen, Levermore and Liu [10 and Jin and Xin [15, we follow here a similar approach to the work of Suliciu [19] (see also [12], 4], [7, 1], 2] ...). We focus here on the particular system (1)-(4) under consideration in this paper. It consists in replacing the non linear pressure law $p(\tau)$ in the original model by a new independent variable evolving according to its own equation. More precisely, we propose to consider the following non linear first order system with singular perturbation :

$$
\left\{\begin{array}{l}
\partial_{t} \tau-\partial_{x} u=0, \\
\partial_{t} u+\partial_{x} \pi=0, \\
\partial_{t} \mathcal{T}=\frac{1}{\epsilon}(\tau-\mathcal{T}),
\end{array}\right.
$$

with the following closure equation

$$
\pi=\pi(\mathcal{T})=p(\mathcal{T})+a^{2}(\mathcal{T}-\tau) .
$$


From this definition, we clearly recover the original system (1) in the asymptotic regime $\epsilon \rightarrow 0$ since we get $\mathcal{T}=\tau$. At least formally and for small positive values of $\epsilon$, the solutions of the relaxation system (24) are thus expected to be very close to the solutions of the equilibrium system (11). We refer for instance the reader to [7] for a rigorous statement of this convergence result under the wellknown assumption that information propagates faster in (24) than in (11) (see Chen, Levermore and Liu [10]). As will be seen hereafter, this sub-characteristic condition writes here

$$
a^{2}>\max _{\tau}-p^{\prime}(\tau)
$$

for all the $\tau$ under consideration. For the sake of simplicity, let us introduce the condensed notation $\mathbf{v}^{r}=(\tau, u, \mathcal{T})^{t}$ together with the natural phase space :

$$
\Omega^{r}=\left\{\mathbf{v}^{r}=(\tau, u, \mathcal{T})^{t} \in \mathbb{R}^{3}, \tau>0, \mathcal{T}>0\right\}
$$

The next lemma, the proof of which is left to the reader, motivates the interest of the relaxation system.

Lemma 2.1. Let be given $a>0$. Then the first order system extracted from 24) is hyperbolic over $\Omega^{r}$ with the following distinct eigenvalues :

$$
\lambda^{(-1, r)}=-a, \quad \lambda^{(0, r)}=0, \quad \lambda^{(1, r)}=a,
$$

and all the characteristic fields are linearly degenerate.

As a consequence of the linear degeneracy of the characteristic fields (see [14), the exact Riemann solution $(x, t) \rightarrow \mathbf{v}^{r}\left(x / t ; \mathbf{v}_{L}^{r}, \mathbf{v}_{R}^{r}\right)$ to

$$
\left\{\begin{array}{l}
\partial_{t} \tau-\partial_{x} u=0 \\
\partial_{t} u+\partial_{x} \pi=0 \\
\partial_{t} \mathcal{T}=0
\end{array}\right.
$$

with initial condition

$$
\mathbf{v}(x, t=0)=\left\{\begin{array}{lll}
\mathbf{v}_{L}^{r} & \text { if } & x<0 \\
\mathbf{v}_{R}^{r} & \text { if } & x>0
\end{array}\right.
$$

is explicitly known (see for instance 4] or [1]) for all $\mathbf{v}_{L}^{r}$ and $\mathbf{v}_{R}^{r}$ in $\Omega^{r}$. More precisely, we have the following lemma :

Lemma 2.2. Let be given $\mathbf{v}_{L}^{r}$ and $\mathbf{v}_{R}^{r}$ in $\Omega^{r}$ at equilibrium, i.e such that $\mathcal{T}_{L}=\tau_{L}$ and $\mathcal{T}_{R}=\tau_{R}$, and $a>0$. Then, the Riemann solution $(x, t) \rightarrow \mathbf{v}^{r}\left(x / t ; \mathbf{v}_{L}^{r}, \mathbf{v}_{R}^{r}\right)$ is made of four constant states separated by three contact discontinuities propagating with velocities $\lambda^{(-1, r)}=-a, \lambda^{(0, r)}=0$ and $\lambda^{(1, r)}=a$, namely

$$
\mathbf{v}^{r}\left(x / t ; \mathbf{v}_{L}^{r}, \mathbf{v}_{R}^{r}\right)=\left\{\begin{aligned}
\mathbf{v}_{L}^{r} & \text { if } \frac{x}{t}<-a, \\
\mathbf{v}_{L *}^{r} & \text { if }-a<\frac{x}{t}<0 \\
\mathbf{v}_{R *}^{r} & \text { if } 0<\frac{x}{t}<a \\
\mathbf{v}_{R}^{r} & \text { if } \frac{x}{t}>a
\end{aligned}\right.
$$


The intermediate states $\mathbf{v}_{L *}^{r}$ and $\mathbf{v}_{R *}^{r}$ are defined from :

$$
\left\{\begin{array}{l}
u_{L *}=u_{R *}=u_{*}:=\frac{1}{2}\left(u_{L}+u_{R}\right)-\frac{1}{2 a}\left(p_{R}-p_{L}\right), \\
\pi_{L *}=\pi_{R *}=\pi_{*}:=\frac{1}{2}\left(p_{L}+p_{R}\right)-\frac{a}{2}\left(u_{R}-u_{L}\right),
\end{array}\right.
$$

and

$$
\begin{aligned}
\tau_{L *}=\tau_{L}+\frac{u_{*}-u_{L}}{a}, & \tau_{R *}=\tau_{R}-\frac{u_{*}-u_{R}}{a}, \\
\mathcal{T}_{L *}=\tau_{L}, & \mathcal{T}_{R *}=\tau_{R} .
\end{aligned}
$$

To conclude this section, the proposed approximate Riemann solver for (12) then naturally corresponds to the choice $l=3$ and

$$
\tilde{\mathbf{v}}\left(\frac{x}{t} ; \mathbf{v}_{L}, \mathbf{v}_{R}\right)= \begin{cases}\mathbf{v}_{L}, & \frac{x}{t}<\lambda^{(-1, r)}=-a, \\ \mathbf{v}_{L *}=\mathbf{v}\left(\mathbf{v}_{L *}^{r}\right), & -a<\frac{x}{t}<\lambda^{(0, r)}=0, \\ \mathbf{v}_{R *}=\mathbf{v}\left(\mathbf{v}_{R *}^{r}\right), & 0<\frac{x}{t}<\lambda^{(1, r)}=a, \\ \mathbf{v}_{R}, & \frac{x}{t}>a,\end{cases}
$$

where we have set

$$
\mathbf{v}\left(\mathbf{v}^{r}\right)=\mathbf{v}\left((\tau, u, \mathcal{T})^{t}\right)=(\tau, u)^{t} .
$$

The typical structure is then given on Figure 3 below. It can be easily proved

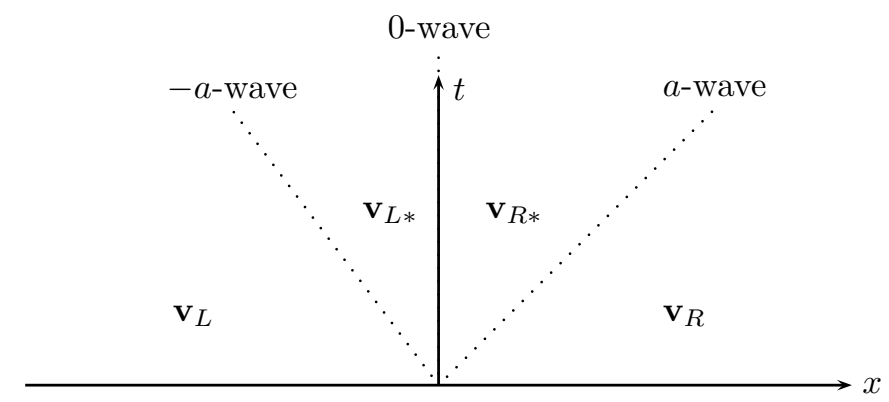

Figure 3: Typical wave structure of a classical relaxation approximate Riemann solution of (12).

that the consistency relation (18) is satisfied. The consistency with the integral form of the entropy inequality (8) is also valid under the CFL condition (17) and provided that the constant $a$ is carefully chosen, more precisely if

$$
a^{2}>\max _{\tau \in\left\lfloor\tau_{L}, \tau_{L *}, \tau_{R *}, \tau_{R}\right\rceil}-p^{\prime}(\tau) .
$$

We refer again for instance the reader to [4, 1], [7]. However, let's keep in mind that this consistency property will be proved in a more general context in the next sections. Let us just mention for the moment that an important quantity 
for such a proof and for the relaxation system (24) is the so-called relaxation specific total energy $\Sigma\left(\mathbf{v}^{r}\right)$ defined by:

$$
\Sigma\left(\mathbf{v}^{r}\right)=\frac{u^{2}}{2}+e(\mathcal{T})+\frac{\pi^{2}-p^{2}(\mathcal{T})}{2 a^{2}} .
$$

It plays the role of a relaxation entropy which coincides at equlibrium $\mathcal{T}=\tau$ with the entropy $\mathcal{U}$ defined by (5). By the chain rule and for smooth solutions of (24), we easily get:

$$
\partial_{t} \Sigma\left(\mathbf{v}^{r}\right)+\partial_{x} \pi\left(\mathbf{v}^{r}\right) u=-\frac{1}{\epsilon}\left(a^{2}+p^{\prime}(\mathcal{T})\right)(\mathcal{T}-\tau)^{2},
$$

the right-hand side being negative under the sub-characteristic condition (26) (the relaxation entropy is dissipated by the relaxation procedure). Then, the proposed relaxation process is entropy consistent in the sense of [10].

\section{Generalized Suliciu Relaxation ARS}

In order to motivate the introduction of a generalized Suliciu relaxation ARS, let us observe that if the initial data is such that $\mathbf{v}_{L}$ and $\mathbf{v}_{R}$ can be joined by an isolated admissible shock wave propagating with velocity $s \neq 0\left(s=s\left(\mathbf{v}_{L}, \mathbf{v}_{R}\right)\right)$, there is no chance for the classical Suliciu relaxation ARS to be exact. Indeed, the sub-characteristic condition imposes in particular $-a<s<a$. Note also that this relaxation ARS satisfies $\mathcal{T}_{L *}=\tau_{L}$ and $\mathcal{T}_{R *}=\tau_{R}$ which is not expected for an isolated shock wave since the discontinuity between the left and right states should occur across a wave propagating with velocity $s$ and not across an artificial stationary wave. In order to overcome this shortcoming, we propose to introduce an additional wave in this Approximate Riemann Solver, with a speed of propagation to be defined and denoted by $\sigma$. Naturally, one of our objectives will be to make $\sigma$ coincide with $s$ in the case $\mathbf{v}_{L}$ and $\mathbf{v}_{R}$ can be joined by an admissible shock wave propagating with velocity $s$. The typical wave structure of this new ARS is represented on Figures 4 and 5 below, for the specific choices $\sigma>0$ and $\sigma<0$ respectively. The original idea is in fact to introduce a new intermediate state in the solution and to impose a relevant jump condition for $\mathcal{T}$ across this new wave. This jump condition will in fact dictate the definition of the intermediate states $\mathbf{v}_{1}, \mathbf{v}_{2}$ and $\mathbf{v}_{3}$ on Figures 4 and 5 More precisely, being given $\mathbf{v}_{L}^{r}$ and $\mathbf{v}_{R}^{r}$ in $\Omega^{r}$ at equilibrium, i.e such that $\mathcal{T}_{L}=\tau_{L}$ and $\mathcal{T}_{R}=\tau_{R}$, and setting $\mathbf{v}_{L}=\mathbf{v}\left(\mathbf{v}_{L}^{r}\right)$ and $\mathbf{v}_{R}=\mathbf{v}\left(\mathbf{v}_{R}^{r}\right)$ as in (31), the new ARS will be given by the exact Riemann solver of the following modified relaxation system

$$
\left\{\begin{array}{l}
\partial_{t} \tau-\partial_{x} u=0 \\
\partial_{t} u+\partial_{x} \pi=0 \\
\partial_{t} \mathcal{T}=\mathcal{M}\left(\mathbf{v}_{L}, \mathbf{v}_{R}, \theta\right) \delta_{x=\sigma t},
\end{array}\right.
$$

where for some real parameter $\theta \in[0,1]$, the weight $\mathcal{M}\left(\mathbf{v}_{L}, \mathbf{v}_{R}, \theta\right)$ of the Dirac measure concentrated on the new discontinuity $x=\sigma t$ with $\sigma=\sigma\left(\mathbf{v}_{L}, \mathbf{v}_{R}\right)$ will be such that

$$
\mathcal{T}_{2}(\theta) \in\left\lfloor\mathcal{T}_{L}, \mathcal{T}_{R}\right\rceil
$$


Here $\mathcal{T}_{2}(\theta)$ is defined as a nonlinear function of the real parameter $\theta$, solving under a relevant sub-characteristic condition given hereafter,

$$
p\left(\mathcal{T}_{2}(\theta)\right)+a^{2} \mathcal{T}_{2}(\theta)=\mathcal{I}_{2}(\theta),
$$

with

$$
\mathcal{I}_{2}(\theta)= \begin{cases}(1-\theta) \mathcal{I}_{L}+\theta \mathcal{I}_{R}, & \sigma<0 \\ \theta \mathcal{I}_{L}+(1-\theta) \mathcal{I}_{R}, & \sigma>0 .\end{cases}
$$

Here and with clear notations, $\mathcal{I}_{k}=p\left(\mathcal{T}_{k}\right)+a^{2} \mathcal{T}_{k}, k=L, R$. We will still have by construction $\mathcal{T}_{1}=\tau_{L}$ and $\mathcal{T}_{3}=\tau_{R}$. The free parameter $\theta \in[0,1]$ will be defined hereafter in order to satisfy the entropy inequality (20) with $(\eta, q)=(\mathcal{U}, \mathcal{F})$. Let us observe from (37) that we will recover the classical Suliciu relaxation ARS of the previous section for the choice $\theta=0$, while the choice $\theta=1$ will restore the case of an admissible isolated shock wave between $\mathbf{v}_{L}$ and $\mathbf{v}_{R}$ and propagating with velocity $s$, and will naturally lead to $\mathbf{v}_{1}=\mathbf{v}_{2}=\mathbf{v}_{L}, \mathbf{v}_{3}=\mathbf{v}_{R}$ and $s=\sigma$ if $s>0$, and $\mathbf{v}_{1}=\mathbf{v}_{L}, \mathbf{v}_{2}=\mathbf{v}_{3}=\mathbf{v}_{R}$ and $s=\sigma$ if $s<0$. Let us underline at this stage that in practice, only the definition of $I_{2}(\theta)$ will matter in the sense that the explicit knowledge of $\mathcal{T}_{2}(\theta)$ can be completely bypassed in the finite volume formulation. Its interest relies on the non linear entropy analysis we intend to perform.

\subsection{Definition of $\sigma$}

Before giving the proposed ARS, let us be more precise on the definition of $\sigma$. This speed of propagation of the additional wave plays in some sense the role of a prediction for the speed of propagation $s$ of an isolated shock wave joining two states $\mathbf{v}_{-}$and $\mathbf{v}_{+}$in the exact Riemann solution associated with the initial states $\mathbf{v}_{L}$ and $\mathbf{v}_{R}$. By the well-known Rankine-Hugoniot relations, we have

$$
\left\{\begin{array}{c}
s=-\frac{u_{+}-u_{-}}{\tau_{+}-\tau_{-}}, \\
s=\frac{p_{+}-p_{-}}{u_{+}-u_{-}},
\end{array}\right.
$$

with $\tau_{+}<\tau_{-}$and $s<0$ for an admissible shock associated with the first eigenvalue $\lambda^{(-1)}$ and $\tau_{+}>\tau_{-}$and $s>0$ for an admissible shock associated with $\lambda^{(1)}$. Recall that the latter relations are equivalent to the validity of the entropy inequality (47) across the discontinuity, that is to

$$
-s\left(\mathcal{U}_{R}-\mathcal{U}_{L}\right)+\left(\mathcal{F}_{R}-\mathcal{F}_{L}\right) \leq 0,
$$

see for instance [14. Note also that the first two relations also give

$$
s^{2}=-\frac{p_{+}-p_{-}}{\tau_{+}-\tau_{-}},
$$

this relation involving only the variable $\tau$. The right-hand side of (38) is always positive since $p^{\prime}(\tau)<0$ for all $\tau$ by assumption. As a natural and generic 
approximation of $s$, we propose (with a little abuse in the notations)

$$
\sigma=\sigma\left(\mathbf{v}_{L}, \mathbf{v}_{R}\right)=\sigma\left(\tau_{L}, \tau_{R}\right)=\left\{\begin{array}{ccc}
-\sqrt{-\frac{p_{R}-p_{L}}{\tau_{R}-\tau_{L}}} & \text { if } & \tau_{R}<\tau_{L}, \\
\sqrt{-\frac{p_{R}-p_{L}}{\tau_{R}-\tau_{L}}} & \text { if } & \tau_{R}>\tau_{L} .
\end{array}\right.
$$

As expected, this prediction is of course exact when $\mathbf{v}_{L}$ and $\mathbf{v}_{R}$ can actually be joined by an admissible discontinuity. Note also that it is actually well-defined even in the asymptotic regime $\tau_{L}=\tau_{R}$ since

$$
\lim _{\tau_{R} \rightarrow \tau_{L}^{-}} \sigma=-\sqrt{-p^{\prime}\left(\tau_{L}\right)}
$$

and

$$
\lim _{\tau_{R} \rightarrow \tau_{L}^{+}} \sigma=+\sqrt{-p^{\prime}\left(\tau_{L}\right)} .
$$

The apparent defect in continuity will be discussed in more details but notice from now on that it will not prevent the proposed ARS to be Lipschitzcontinuous.

The next two sections define the proposed ARS in both situations $\sigma>0$ and $\sigma<0$.

\subsection{The case $\sigma>0$}

We turn in this section to the definition of the intermediate states $\mathbf{v}_{1}, \mathbf{v}_{2}$ and $\mathbf{v}_{3}$ in the case $\sigma>0$, see Figure 4 Each state containing three components, nine

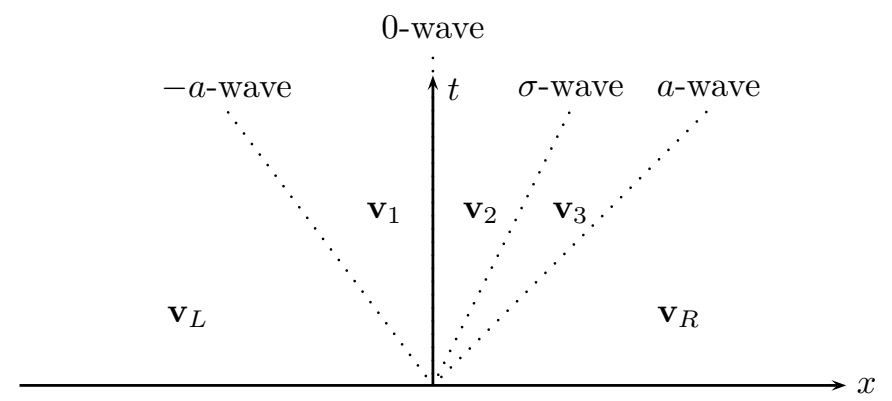

Figure 4: Typical wave structure of the generalized Suliciu relaxation ARS in the case $\sigma>0$.

relations are expected. As motivated in the previous section, we first impose the consistency relations (18) associated with the first two equations of (35), 
which gives here

$$
\left\{\begin{array}{l}
\left(u_{L}-u_{R}\right)=-a\left(\tau_{1}-\tau_{L}\right)+\sigma\left(\tau_{3}-\tau_{2}\right)+a\left(\tau_{R}-\tau_{3}\right), \\
\pi_{R}-\pi_{L}=-a\left(u_{1}-u_{L}\right)+\sigma\left(u_{3}-u_{2}\right)+a\left(u_{R}-u_{3}\right),
\end{array}\right.
$$

where $\pi_{L}=p_{L}$ and $\pi_{R}=p_{R}$ since $\mathbf{v}_{L}^{r}$ and $\mathbf{v}_{R}^{r}$ are assumed to be at equilibrium $\left(\mathcal{T}_{L}=\tau_{L}\right.$ and $\left.\mathcal{T}_{R}=\tau_{R}\right)$. Then, we make the natural choice of imposing the Rankine-Hugoniot relations associated with the mass conservation (i.e. the first equation in (35) ) across each wave of the ARS. We get

$$
\left\{\begin{array}{l}
u_{L}-a \tau_{L}=u_{1}-a \tau_{1} \\
u_{1}=u_{2} \\
u_{2}+\sigma \tau_{2}=u_{3}+\sigma \tau_{3} \\
u_{R}+a \tau_{R}=u_{3}+a \tau_{3}
\end{array}\right.
$$

Note however that (41) provides only three additional independent relations since the first equation of (40) is a linear combination of the four equations in (411). Then, four equations are still missing.

Considering now the Rankine-Hugoniot relations associated with the momentum conservation (i.e. the second equation in (35)) across each wave of the ARS leads to

$$
\left\{\begin{array}{l}
a u_{L}+\pi_{L}=a u_{1}+\pi_{1}, \\
\pi_{1}=\pi_{2}, \\
\sigma u_{2}-\pi_{2}=\sigma u_{3}-\pi_{3}, \\
a u_{R}-\pi_{R}=a u_{3}-\pi_{3} .
\end{array}\right.
$$

Here again, the sum of the four equations in (42) gives the second equation in (40), so that one equation is still missing. Note in passing that the first (respectively last) equations of (41) and (42) imply that $\mathcal{I}=\pi+a^{2} \tau$ is constant across the first (resp. last) wave, that is

$$
\left\{\begin{array}{l}
\mathcal{I}_{1}=\mathcal{I}_{L}, \\
\mathcal{I}_{R}=\mathcal{I}_{3},
\end{array}\right.
$$

or equivalently (still under a relevant sub-characteristic condition given hereafter)

$$
\left\{\begin{array}{l}
\mathcal{T}_{1}=\mathcal{T}_{L} \\
\mathcal{T}_{R}=\mathcal{T}_{3}
\end{array}\right.
$$

thanks to (36). At last, the system is closed by considering the RankineHugoniot relation associated with the last equation in (35) and the $\sigma$-wave, which writes here

$$
-\sigma\left(\mathcal{T}_{3}-\mathcal{T}_{2}\right)=\mathcal{M}\left(\mathbf{v}_{L}, \mathbf{v}_{R}, \theta\right)
$$

where $\mathcal{M}\left(\mathbf{v}_{L}, \mathbf{v}_{R}, \theta\right)$ is such that

$$
\mathcal{I}_{2}=(1-\theta) \mathcal{I}_{R}+\theta \mathcal{I}_{L}, \quad \theta \in[0,1] .
$$

Note in particular that for $\theta=0$, we have $\mathcal{I}_{2}=\mathcal{I}_{R}$ which is expected. 
Solving (41)-(42)-(45). Thus, one has to solve the nine by nine linear system (41)-(42)- (45) in order to determine the intermediate states of our ARS. Let us first evaluate the jumps of $\tau, u$ and $\pi$ across the $\sigma$-wave. The last equation of (43) together with (45) first gives

$$
\pi_{3}-\pi_{2}=\theta\left(\mathcal{I}_{R}-\mathcal{I}_{L}\right)-a^{2}\left(\tau_{3}-\tau_{2}\right)
$$

with, thanks to mass and momentum conservation across the $\sigma$-wave,

$$
\sigma\left(\tau_{3}-\tau_{2}\right)=-\left(u_{3}-u_{2}\right)=-\frac{\pi_{3}-\pi_{2}}{\sigma} .
$$

Then we get

$$
\pi_{3}-\pi_{2}=\theta\left(\mathcal{I}_{R}-\mathcal{I}_{L}\right)+\frac{a^{2}}{\sigma^{2}}\left(\pi_{3}-\pi_{2}\right),
$$

and straightforwardly

$$
\left\{\begin{array}{l}
\pi_{3}-\pi_{2}=-\frac{\sigma^{2} \theta\left(\mathcal{I}_{R}-\mathcal{I}_{L}\right)}{a^{2}-\sigma^{2}}=-\sigma^{2} \theta\left(\tau_{R}-\tau_{L}\right), \\
u_{3}-u_{2}=-\frac{\sigma \theta\left(\mathcal{I}_{R}-\mathcal{I}_{L}\right)}{a^{2} \overline{\mathcal{F}}^{2}}=-\sigma \theta\left(\tau_{R}-\tau_{L}\right), \\
\tau_{3}-\tau_{2}=\frac{\theta\left(\mathcal{I}_{R}-\overline{\mathcal{I}}_{L}\right)}{a^{2}-\sigma^{2}}=\theta\left(\tau_{R}-\tau_{L}\right),
\end{array}\right.
$$

since $\mathcal{I}_{R}-\mathcal{I}_{L}=\left(a^{2}-\sigma^{2}\right)\left(\tau_{R}-\tau_{L}\right)\left(\right.$ recall that $\mathcal{I}_{R}-\mathcal{I}_{L}=\left(p_{R}-p_{L}\right)+a^{2}\left(\tau_{R}-\right.$ $\left.\tau_{L}\right)$ and $\left.p_{R}-p_{L}=-\sigma^{2}\left(\tau_{R}-\tau_{L}\right)\right)$. As expected, we clearly recover that the jumps of $\tau, u$ and $\pi$ are trivial when $\theta$ goes to zero and the classical Suliciu relaxation solver is recovered. Let us then evaluate the pressure and velocity associated with $\mathbf{v}_{2}$ and $\mathbf{v}_{3}$. Since the pressure and velocity are constant across the 0 -wave, the first and last equations of (42) give

$$
\left\{\begin{array}{l}
a u_{L}+\pi_{L}=a u_{2}+\pi_{2}, \\
a u_{R}-\pi_{R}=a u_{3}-\pi_{3},
\end{array}\right.
$$

or equivalently, using (46),

$$
\left\{\begin{array}{l}
\pi_{3}+\pi_{2}=2 \pi_{*}-\frac{a \sigma \theta\left(\mathcal{I}_{R}-\mathcal{I}_{L}\right)}{a^{2}-\sigma^{2}}=2 \pi_{*}-a \sigma \theta\left(\tau_{R}-\tau_{L}\right), \\
u_{3}+u_{2}=2 u_{*}-\frac{\sigma^{2} \theta\left(\mathcal{I}_{R}-\mathcal{I}_{L}\right)}{a\left(a^{2}-\sigma^{2}\right)}=2 u_{*}-\frac{\sigma^{2} \theta}{a}\left(\tau_{R}-\tau_{L}\right),
\end{array}\right.
$$

where we have set

$$
\left\{\begin{array}{l}
u_{*}=\frac{1}{2}\left(u_{L}+u_{R}\right)-\frac{1}{2 a}\left(\pi_{R}-\pi_{L}\right), \\
\pi_{*}=\frac{1}{2}\left(\pi_{L}+\pi_{R}\right)-\frac{a}{2}\left(u_{R}-u_{L}\right) .
\end{array}\right.
$$

Observe that these values correspond to the intermediate values of the velocity and pressure in the classical Suliciu relaxation Approximate Riemann Solver 
proposed in section 2.3.2 Combining (46) and (47) easily gives

$$
\left\{\begin{array}{l}
\pi_{2}=\pi_{*}-\frac{\sigma \theta\left(\mathcal{I}_{R}-\mathcal{I}_{L}\right)}{2(a+\sigma)}=\pi_{*}-\frac{\sigma \theta}{2}(a-\sigma)\left(\tau_{R}-\tau_{L}\right), \\
\pi_{3}=\pi_{*}-\frac{\sigma \theta\left(\mathcal{I}_{R}-\mathcal{I}_{L}\right)}{2(a-\sigma)}=\pi_{*}-\frac{\sigma \theta}{2}(a+\sigma)\left(\tau_{R}-\tau_{L}\right), \\
u_{2}=u_{*}+\frac{\sigma \theta\left(\mathcal{I}_{R}-\mathcal{I}_{L}\right)}{2 a(a+\sigma)}=u_{*}+\frac{\sigma \theta}{2 a}(a-\sigma)\left(\tau_{R}-\tau_{L}\right), \\
u_{3}=u_{*}-\frac{\sigma \theta\left(\mathcal{I}_{R}-\mathcal{I}_{L}\right)}{2 a(a-\sigma)}=u_{*}-\frac{\sigma \theta}{2 a}(a+\sigma)\left(\tau_{R}-\tau_{L}\right),
\end{array}\right.
$$

while the specific volumes follow from the mass conservation across the $a$-wave :

$$
\left\{\begin{array}{l}
\tau_{3}=\tau_{R}+\frac{1}{a}\left(u_{R}-u_{3}\right), \\
\tau_{2}=\tau_{3}-\frac{\theta\left(\mathcal{I}_{R}-\mathcal{I}_{L}\right)}{a^{2}-\sigma^{2}}=\tau_{3}-\theta\left(\tau_{R}-\tau_{L}\right) .
\end{array}\right.
$$

In particular, we note that when $\theta$ equals zero we recover the intermediate values associated with the classical Suliciu relaxation approach. On the other hand, the mass conservation across the $-a$-wave allows to determine the specific volume of $\mathbf{v}_{1}$ by

$$
\tau_{1}=\tau_{L}+\frac{1}{a}\left(u_{1}-u_{L}\right),
$$

which completes the calculation of the three intermediate states since

$$
\left\{\begin{array}{l}
u_{1}=u_{2}, \\
\pi_{1}=\pi_{2} .
\end{array}\right.
$$

\subsection{The general case}

The case $\sigma<0$ can be treated following exactly the same way but considering the wave pattern given on Figure 5

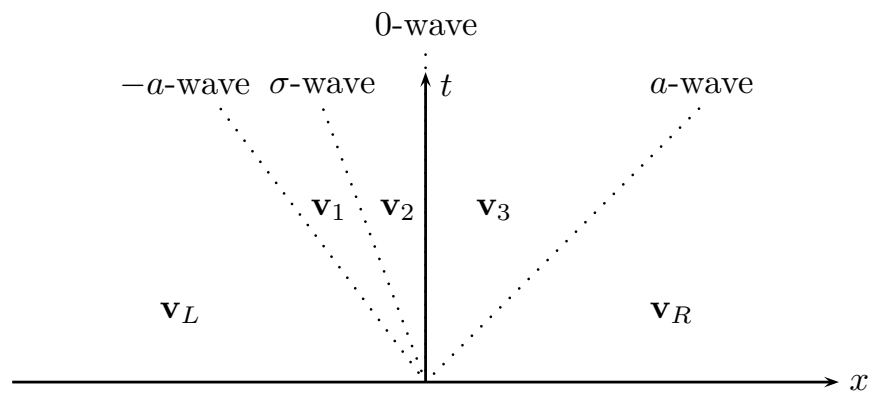

Figure 5: Typical wave structure of the generalized Suliciu relaxation ARS in the case $\sigma<0$. 
Actually, we can take advantage of the underlying Galilean symmetry to infer the required formulae from the ones derived in the setting of a positive wave speed $\sigma>0$. Galilean symmetry is achieved when reversing the $x$-axis and exchanging the sign of all velocities. Namely being given a pair of states $\left(\mathbf{v}_{L}^{r}, \mathbf{v}_{R}^{r}\right)$ with associated wave speed $\sigma<0$, we first define $\hat{\mathbf{v}}_{L}^{r}$ (respectively $\left.\hat{\mathbf{v}}_{R}^{r}\right)$ from $\mathbf{v}_{L}^{r}\left(\right.$ resp. $\left.\mathbf{v}_{R}^{r}\right)$ with velocity $\hat{u}_{L}=-u_{L}\left(\right.$ resp. $\left.\hat{u}_{R}=-u_{R}\right)$, all the other data being kept unchanged. We then solve the generalized Riemann solution which we denote $\hat{\omega}\left(x, t ; \hat{\mathbf{v}}_{R}^{r}, \hat{\mathbf{v}}_{L}^{r},-\sigma\right)$ when changing the speed sign of the additional wave $\hat{\sigma}=-\sigma$. Let us stress that by Galilean symmetry, the role of the modified left and right states and the sign of the additional wave speed $\hat{\sigma}=-\sigma>0$ have been reversed. The self-similar solution coming with the original pair $\left(\mathbf{v}_{L}^{r}, \mathbf{v}_{R}^{r}\right)$, denoted by $\omega\left(x, t ; \mathbf{v}_{L}^{r}, \mathbf{v}_{R}^{r},+\sigma\right)$, is then recovered when reversing the $x$-axis and the sign of all the velocities in the formulae defining $\hat{\omega}\left(x, t ; \hat{\mathbf{v}}_{R}^{r}, \hat{\mathbf{v}}_{L}^{r},-\sigma\right)$, the other quantities being kept unchanged, except $\tau_{2}$ and $\mathcal{I}_{2}(\theta)$.

In order to gather the definitions of the intermediate states in the same formalism in both cases $\sigma<0$ and $\sigma>0$, we propose to use the subscript (respectively + ) for the left (resp. right) state of the $\sigma$-wave, instead of ${ }_{2}$ in the case $\sigma>0$ and ${ }_{1}$ in the case $\sigma<0$ (resp. ${ }_{3}$ in the case $\sigma>0$ and ${ }_{2}$ in the case $\sigma<0)$. It is then easily shown that we have the following definitions for the components of the intermediate states, the calculations being left to the reader :

$$
\left\{\begin{aligned}
u_{-}= & u_{*}+\frac{\sigma \theta\left(\mathcal{I}_{R}-\mathcal{I}_{L}\right)}{2 a(a+\sigma)}=u_{*}+\frac{\sigma \theta}{2 a}(a-\sigma)\left(\tau_{R}-\tau_{L}\right), \\
u_{+}= & u_{*}-\frac{\sigma \theta\left(\mathcal{I}_{R}-\mathcal{I}_{L}\right)}{2 a(a-\sigma)}=u_{*}-\frac{\sigma \theta}{2 a}(a+\sigma)\left(\tau_{R}-\tau_{L}\right), \\
\tau_{1}= & \tau_{L}+\frac{1}{q}\left(u_{-}-u_{L}\right), \\
\tau_{3}= & \tau_{R}+\frac{1}{a}\left(u_{R}-u_{+}\right), \\
& \left\{\begin{array}{c}
\tau_{1}+\frac{\sigma \theta\left(\mathcal{I}_{R}-\mathcal{I}_{L}\right)}{\sigma\left(a^{2}-\sigma^{2}\right)}=\tau_{1}+\theta\left(\tau_{R}-\tau_{L}\right), \quad \sigma<0, \\
\tau_{3}-\frac{\sigma \theta\left(\mathcal{I}_{R}-\mathcal{I}_{L}\right)}{\sigma\left(a^{2}-\sigma^{2}\right)}=\tau_{3}-\theta\left(\tau_{R}-\tau_{L}\right), \quad \sigma>0,
\end{array}\right. \\
\pi_{-}= & \pi_{*}-\frac{\sigma \theta\left(\mathcal{I}_{R}-\mathcal{I}_{L}\right)}{2(a+\sigma)}=\pi_{*}-\frac{\sigma \theta}{2}(a-\sigma)\left(\tau_{R}-\tau_{L}\right), \\
\pi_{+}= & \pi_{*}-\frac{\sigma \theta\left(\mathcal{I}_{R}-\mathcal{I}_{L}\right)}{2(a-\sigma)}=\pi_{*}-\frac{\sigma \theta}{2}(a+\sigma)\left(\tau_{R}-\tau_{L}\right),
\end{aligned}\right.
$$

with

$$
\left\{\begin{array}{l}
\mathcal{I}_{1}=\mathcal{I}_{L}, \\
\mathcal{I}_{2}(\theta)= \begin{cases}(1-\theta) \mathcal{I}_{L}+\theta \mathcal{I}_{R}=\mathcal{I}_{L}+\theta\left(\mathcal{I}_{R}-\mathcal{I}_{L}\right), & \sigma<0, \\
(1-\theta) \mathcal{I}_{R}+\theta \mathcal{I}_{L}=\mathcal{I}_{R}-\theta\left(\mathcal{I}_{R}-\mathcal{I}_{L}\right), & \sigma>0,\end{cases} \\
\mathcal{I}_{3}=\mathcal{I}_{R} .
\end{array}\right.
$$

Observe that we have expressed the values of the relaxation pressure $\pi$ everywhere within the fan instead of the underlying values of the relaxation specific 
volume $\mathcal{T}$. In fact, only the values of $(\tau, u, \pi)$ enter the definition of the forthcoming numerical flux function. Notice that all these values linearly depend on the real parameter $\theta$, and are thus readily defined as soon as $\theta$ is conveniently prescribed. As will be seen in the forthcoming sections, the non linear function $\mathcal{T}_{2}(\theta)$ is in fact implicitly defined according to (36) so as to yield some explicit bound for $\theta$ from a non linear entropy analysis.

\subsection{Basic properties}

We conclude this section by establishing some basic properties that will be useful in the forthcoming developments.

Lemma 3.1. Under the sub-characteristic condition

$$
a^{2}>\max _{\tau \in\left\lfloor\tau_{L}, \tau_{R}\right\rceil}-p^{\prime}(\tau)
$$

we have

(i) $\sigma\left(\mathcal{I}_{R}-\mathcal{I}_{L}\right) \geq 0$ (and then $\sigma \theta\left(\mathcal{I}_{R}-\mathcal{I}_{L}\right) \geq 0$ ),

(ii) $\tau_{1} \geq \tau_{L *}>0$

(iii) $\tau_{3} \geq \tau_{R *}>0$

(iv) $\tau_{2} \leq \tau_{L *}$ if $\sigma<0$,

(v) $\tau_{2} \leq \tau_{R *}$ if $\sigma>0$,

where $\tau_{L *}$ and $\tau_{R *}$ are defined in 29). In addition and provided that $\theta$ satisfies

$$
\begin{array}{ll}
\sigma \theta\left(\mathcal{I}_{R}-\mathcal{I}_{L}\right) \leq \frac{a^{2}|\sigma|(a+|\sigma|)}{a+|\sigma| / 2}(1-\alpha) \tau_{L *} & \text { if } \quad \sigma<0, \\
\sigma \theta\left(\mathcal{I}_{R}-\mathcal{I}_{L}\right) \leq \frac{a^{2}|\sigma|(a+|\sigma|)}{a+|\sigma| / 2}(1-\alpha) \tau_{R *} & \text { if } \quad \sigma>0,
\end{array}
$$

we have for all $\alpha \in(0,1)$

(vi) $\tau_{2} \geq \alpha \tau_{L *}$ if $\sigma<0$

(vii) $\tau_{2} \geq \alpha \tau_{R *}$ if $\sigma>0$.

Proof. Let us first recall that $\theta \in[0,1]$ and $\sigma$ is defined by (39).

(i) We clearly have by definition of $\mathcal{I}=\mathcal{I}(\tau)$

$$
\sigma\left(\mathcal{I}_{R}-\mathcal{I}_{L}\right)=\sigma\left(p\left(\tau_{R}\right)-p\left(\tau_{L}\right)+a^{2}\left(\tau_{R}-\tau_{L}\right)\right)=\sigma\left(\tau_{R}-\tau_{L}\right)\left(a^{2}+p^{\prime}(\xi)\right)
$$

for some $\xi$ in the interval $\left\lfloor\tau_{L}, \tau_{R}\right\rceil$. Which clearly gives the conclusion under the condition (52) and since $\sigma\left(\tau_{R}-\tau_{L}\right) \geq 0$ by (39).

(ii) We have by (50)

$$
\tau_{1}=\tau_{L}+\frac{u_{*}-u_{L}}{a}+\frac{\sigma \theta\left(\mathcal{I}_{R}-\mathcal{I}_{L}\right)}{2 a^{2}(a+\sigma)},
$$

that is by 29

$$
\tau_{1}=\tau_{L *}+\frac{\sigma \theta\left(\mathcal{I}_{R}-\mathcal{I}_{L}\right)}{2 a^{2}(a+\sigma)}
$$


Under the condition (52), we clearly have $(a+\sigma)>0$ so that $\tau_{1} \geq \tau_{L *}$ by $(i)$.

(iii) We have by (50)

$$
\tau_{3}=\tau_{R}+\frac{u_{R}-u_{*}}{a}+\frac{\sigma \theta\left(\mathcal{I}_{R}-\mathcal{I}_{L}\right)}{2 a^{2}(a-\sigma)},
$$

that is by (29)

$$
\tau_{3}=\tau_{R *}+\frac{\sigma \theta\left(\mathcal{I}_{R}-\mathcal{I}_{L}\right)}{2 a^{2}(a-\sigma)}
$$

Under the condition (52), we clearly have $(a-\sigma)>0$ so that $\tau_{3} \geq \tau_{R *}$ by $(i)$.

(iv) We have by (50)

$$
\tau_{2}=\tau_{1}+\frac{\sigma \theta\left(\mathcal{I}_{R}-\mathcal{I}_{L}\right)}{\sigma\left(a^{2}-\sigma^{2}\right)}
$$

that is

$$
\tau_{2}=\tau_{L *}+\frac{\sigma \theta\left(\mathcal{I}_{R}-\mathcal{I}_{L}\right)}{2 a^{2}(a+\sigma)}+\frac{\sigma \theta\left(\mathcal{I}_{R}-\mathcal{I}_{L}\right)}{\sigma\left(a^{2}-\sigma^{2}\right)}
$$

or equivalently

$$
\tau_{2}=\tau_{L *}+\frac{\sigma \theta\left(\mathcal{I}_{R}-\mathcal{I}_{L}\right)\left(2 a^{2}+\sigma a-\sigma^{2}\right)}{2 a^{2} \sigma\left(a^{2}-\sigma^{2}\right)}
$$

Under the condition (52), we clearly have $\left(2 a^{2}+\sigma a-\sigma^{2}\right)=2(a+\sigma)(a-\sigma / 2)>0$ and then $\tau_{2} \leq \tau_{L *}$ since here $\sigma<0$.

$(v)$ We have by (50)

$$
\tau_{2}=\tau_{3}-\frac{\sigma \theta\left(\mathcal{I}_{R}-\mathcal{I}_{L}\right)}{\sigma\left(a^{2}-\sigma^{2}\right)}
$$

that is

$$
\tau_{2}=\tau_{R *}+\frac{\sigma \theta\left(\mathcal{I}_{R}-\mathcal{I}_{L}\right)}{2 a^{2}(a-\sigma)}-\frac{\sigma \theta\left(\mathcal{I}_{R}-\mathcal{I}_{L}\right)}{\sigma\left(a^{2}-\sigma^{2}\right)},
$$

or equivalently

$$
\tau_{2}=\tau_{R *}+\frac{\sigma \theta\left(\mathcal{I}_{R}-\mathcal{I}_{L}\right)\left(-2 a^{2}+\sigma a+\sigma^{2}\right)}{2 a^{2} \sigma\left(a^{2}-\sigma^{2}\right)} .
$$

Under the condition (52) and since we have assumed $\sigma>0$, we clearly have $\left(-2 a^{2}+\sigma a-\sigma^{2}\right)=-2 a(a-\sigma)(a+\sigma / 2)<0$ and then $\tau_{2} \leq \tau_{R *}$.

(vi) Let us assume $\sigma<0$. We have

$$
\tau_{2}=\tau_{L *}+\frac{\sigma \theta\left(\mathcal{I}_{R}-\mathcal{I}_{L}\right)\left(2 a^{2}+\sigma a-\sigma^{2}\right)}{2 a^{2} \sigma\left(a^{2}-\sigma^{2}\right)}
$$

so that

$$
\tau_{2} \geq \alpha \tau_{L *} \quad \Longleftrightarrow \sigma \theta\left(\mathcal{I}_{R}-\mathcal{I}_{L}\right)\left(2 a^{2}+\sigma a-\sigma^{2}\right) \leq 2 a^{2}|\sigma|\left(a^{2}-\sigma^{2}\right)(1-\alpha) \tau_{L *}
$$

which gives as expected

$$
\tau_{2} \geq \alpha \tau_{L *} \quad \Longleftrightarrow \quad \sigma \theta\left(\mathcal{I}_{R}-\mathcal{I}_{L}\right) \leq \frac{a^{2}|\sigma|(a+|\sigma|)}{a+|\sigma| / 2}(1-\alpha) \tau_{L *}
$$


(vii) Let us now assume $\sigma>0$. We have

$$
\tau_{2}=\tau_{R *}+\frac{\sigma \theta\left(\mathcal{I}_{R}-\mathcal{I}_{L}\right)\left(-2 a^{2}+\sigma a+\sigma^{2}\right)}{2 a^{2} \sigma\left(a^{2}-\sigma^{2}\right)}
$$

so that

$$
\tau_{2} \geq \alpha \tau_{R *} \quad \Longleftrightarrow \sigma \theta\left(\mathcal{I}_{R}-\mathcal{I}_{L}\right)\left(2 a^{2}-\sigma a-\sigma^{2}\right) \leq 2 a^{2} \sigma\left(a^{2}-\sigma^{2}\right)(1-\alpha) \tau_{R *}
$$

that is

$$
\tau_{2} \geq \alpha \tau_{R *} \quad \Longleftrightarrow \quad \sigma \theta\left(\mathcal{I}_{R}-\mathcal{I}_{L}\right) \leq \frac{a^{2} \sigma(a+\sigma)}{a+\sigma / 2}(1-\alpha) \tau_{R *}
$$

which is the expected inequality.

\section{Properties of the generalized Suliciu Relax- ation ARS}

Aim of this section is to define the free parameter $\theta$ and establish the main properties of the proposed ARS in terms of non linear stability (entropy inequality), continuity and accuracy.

\subsection{Entropy inequality}

Our objective in this section is to prove under a relevant choice of $\theta$ that the proposed ARS satisfies a discrete entropy inequality. Due to Galilean symmetry, we focus ourselves and without restriction to the situation of a positive velocity $\sigma>0$. According to (20), we have to prove that

$\mathcal{F}\left(\mathbf{v}_{R}\right)-\mathcal{F}\left(\mathbf{v}_{L}\right) \leq-a\left(\mathcal{U}\left(\mathbf{v}_{1}\right)-\mathcal{U}\left(\mathbf{v}_{L}\right)\right)+\sigma\left(\mathcal{U}\left(\mathbf{v}_{3}\right)-\mathcal{U}\left(\mathbf{v}_{2}\right)\right)+a\left(\mathcal{U}\left(\mathbf{v}_{R}\right)-\mathcal{U}\left(\mathbf{v}_{3}\right)\right)$,

where $\mathcal{U}$ and $\mathcal{F}$ are definied by $(5)$. Let us introduce the so-called relaxation entropy-entropy flux pair $\left(\Sigma, \mathcal{F}^{r}\right)$ defined by

$$
\Sigma\left(\mathbf{v}^{r}\right)=\frac{u^{2}}{2}+e(\mathcal{T})+\frac{\pi^{2}-p^{2}(\mathcal{T})}{2 a^{2}}, \quad \mathcal{F}^{r}\left(\mathbf{v}^{r}\right)=\pi u .
$$

We then propose to prove the validity of (54) by showing the following set of equalities and inequalities,

$$
\left\{\begin{aligned}
\mathcal{F}\left(\mathbf{v}_{R}\right)-\mathcal{F}^{r}\left(\mathbf{v}_{3}^{r}\right) & =a\left(\mathcal{U}\left(\mathbf{v}_{R}\right)-\Sigma\left(\mathbf{v}_{3}^{r}\right)\right), \\
0 & \leq(a-\sigma)\left(\Sigma\left(\mathbf{v}_{3}^{r}\right)-\mathcal{U}\left(\mathbf{v}_{3}\right)\right), \\
\mathcal{F}^{r}\left(\mathbf{v}_{3}^{r}\right)-\mathcal{F}^{r}\left(\mathbf{v}_{2}^{r}\right) & \leq \sigma\left(\Sigma\left(\mathbf{v}_{3}^{r}\right)-\Sigma\left(\mathbf{v}_{2}^{r}\right)\right), \\
0 & \leq \sigma\left(\Sigma\left(\mathbf{v}_{2}^{r}\right)-\mathcal{U}\left(\mathbf{v}_{2}\right)\right), \\
\mathcal{F}^{r}\left(\mathbf{v}_{2}^{r}\right)-\mathcal{F}^{r}\left(\mathbf{v}_{1}^{r}\right) & =0, \\
0 & \leq \sigma\left(\Sigma\left(\mathbf{v}_{1}^{r}\right)-\mathcal{U}\left(\mathbf{v}_{1}\right)\right), \\
\mathcal{F}^{r}\left(\mathbf{v}_{1}^{r}\right)-\mathcal{F}\left(\mathbf{v}_{L}\right) & =-a\left(\Sigma\left(\mathbf{v}_{1}^{r}\right)-\mathcal{U}\left(\mathbf{v}_{L}\right)\right), \\
0 & \leq(a+\sigma)\left(\Sigma\left(\mathbf{v}_{1}^{r}\right)-\mathcal{U}\left(\mathbf{v}_{1}\right)\right),
\end{aligned}\right.
$$


which by direct summation clearly resumes to a set of sufficient conditions. Let us first focus on the three equalities, which equivalently write as follows

$$
\left\{\begin{aligned}
\mathcal{F}^{r}\left(\mathbf{v}_{R}^{r}\right)-\mathcal{F}^{r}\left(\mathbf{v}_{3}^{r}\right) & =a\left(\Sigma\left(\mathbf{v}_{R}^{r}\right)-\Sigma\left(\mathbf{v}_{3}^{r}\right)\right) \\
\mathcal{F}^{r}\left(\mathbf{v}_{2}^{r}\right) & =\mathcal{F}^{r}\left(\mathbf{v}_{1}^{r}\right) \\
\mathcal{F}^{r}\left(\mathbf{v}_{1}^{r}\right)-\mathcal{F}^{r}\left(\mathbf{v}_{L}\right) & =-a\left(\Sigma\left(\mathbf{v}_{1}^{r}\right)-\Sigma\left(\mathbf{v}_{L}^{r}\right)\right)
\end{aligned}\right.
$$

since the left and right states $\mathbf{v}_{L}^{r}$ and $\mathbf{v}_{R}^{r}$ are at equilibrium, that is $\pi_{k}=$ $p\left(\mathcal{T}_{k}\right)=p\left(\tau_{k}\right), k=L, R$. The second equality clearly holds since $\pi_{2}=\pi_{1}$ and $u_{2}=u_{1}$. Thanks to the definitions (55) and the continuity properties (44), the first and third ones write

$$
\left\{\begin{array}{l}
\pi_{R} u_{R}-\pi_{3} u_{3}=a\left(\frac{1}{2} u_{R}^{2}+\frac{1}{2 a^{2}} \pi_{R}^{2}-\frac{1}{2} u_{3}^{2}-\frac{1}{2 a^{2}} \pi_{3}^{2}\right), \\
\pi_{1} u_{1}-\pi_{L} u_{L}=-a\left(\frac{1}{2} u_{1}^{2}+\frac{1}{2 a^{2}} \pi_{1}^{2}-\frac{1}{2} u_{L}^{2}-\frac{1}{2 a^{2}} \pi_{L}^{2}\right),
\end{array}\right.
$$

or equivalently

$$
\left\{\begin{aligned}
\frac{\left(\pi_{R}-\pi_{3}\right)\left(u_{R}+u_{3}\right)}{2} & +\frac{\left(u_{R}-u_{3}\right)\left(\pi_{R}+\pi_{3}\right)}{2}= \\
a \frac{\left(u_{R}-u_{3}\right)\left(u_{R}+u_{3}\right)}{2} & +\frac{\left(\pi_{R}-\pi_{3}\right)\left(\pi_{R}+\pi_{3}\right)}{2 a} \\
\frac{\left(\pi_{1}-\pi_{L}\right)\left(u_{1}+u_{L}\right)}{2} & +\frac{\left(u_{1}-u_{L}\right)\left(\pi_{1}+\pi_{L}\right)}{2} \\
& = \\
-a \frac{\left(u_{1}-u_{L}\right)\left(u_{1}+u_{L}\right)}{2} & -\frac{\left(\pi_{1}-\pi_{L}\right)\left(\pi_{1}+\pi_{L}\right)}{2 a}
\end{aligned}\right.
$$

which is valid thanks to the first and last equations of (42). Let us now consider the first, third, fifth and last inequalities of (56). Since $-a<0<\sigma<a$, they are equivalent to

$$
\left\{\begin{array}{l}
\mathcal{U}\left(\mathbf{v}_{3}\right) \leq \Sigma\left(\mathbf{v}_{3}^{r}\right) \\
\mathcal{U}\left(\mathbf{v}_{2}\right) \leq \Sigma\left(\mathbf{v}_{2}^{r}\right) \\
\mathcal{U}\left(\mathbf{v}_{1}\right) \leq \Sigma\left(\mathbf{v}_{1}^{r}\right)
\end{array}\right.
$$

and by definition of $\mathcal{U}$ and $\Sigma$ to

$$
\left\{\begin{array}{l}
2 a^{2}\left(e\left(\tau_{3}\right)-e\left(\mathcal{T}_{3}\right)\right) \leq \pi_{3}^{2}-p^{2}\left(\mathcal{T}_{3}\right) \\
2 a^{2}\left(e\left(\tau_{2}\right)-e\left(\mathcal{T}_{2}\right)\right) \leq \pi_{2}^{2}-p^{2}\left(\mathcal{T}_{2}\right) \\
2 a^{2}\left(e\left(\tau_{1}\right)-e\left(\mathcal{T}_{1}\right)\right) \leq \pi_{1}^{2}-p^{2}\left(\mathcal{T}_{1}\right)
\end{array}\right.
$$

Using that $\pi=p(\mathcal{T})+a^{2}(\mathcal{T}-\tau)$, it equivalently recasts as

$$
\left\{\begin{array}{l}
2\left(e\left(\tau_{3}\right)-e\left(\mathcal{T}_{3}\right)\right) \leq a^{2}\left(\mathcal{T}_{3}-\tau_{3}\right)^{2}+2 p\left(\mathcal{T}_{3}\right)\left(\mathcal{T}_{3}-\tau_{3}\right) \\
2\left(e\left(\tau_{2}\right)-e\left(\mathcal{T}_{2}\right)\right) \leq a^{2}\left(\mathcal{T}_{2}-\tau_{2}\right)^{2}+2 p\left(\mathcal{T}_{2}\right)\left(\mathcal{T}_{2}-\tau_{2}\right) \\
2\left(e\left(\tau_{1}\right)-e\left(\mathcal{T}_{1}\right)\right) \leq a^{2}\left(\mathcal{T}_{1}-\tau_{1}\right)^{2}+2 p\left(\mathcal{T}_{1}\right)\left(\mathcal{T}_{1}-\tau_{1}\right)
\end{array}\right.
$$


so that second-order Taylor expansions around $\mathcal{T}$ easily give

$$
\left\{\begin{array}{l}
-p^{\prime}\left(\xi_{3}\right) \leq a^{2}, \\
-p^{\prime}\left(\xi_{2}\right) \leq a^{2}, \\
-p^{\prime}\left(\xi_{1}\right) \leq a^{2},
\end{array}\right.
$$

for some $\xi_{i}$ within the intervals $\left(\min \left(\tau_{i}, \mathcal{T}_{i}\right), \max \left(\tau_{i}, \mathcal{T}_{i}\right)\right)$. Provided that $a$ is such that the sub-characteristic like condition

$$
a^{2}>\min _{\tau \in\left(\tau_{m}, \tau_{M}\right)}-p^{\prime}(\tau)
$$

with, since $\mathcal{T}_{L}=\mathcal{T}_{1}=\tau_{L}$ and $\mathcal{T}_{3}=\mathcal{T}_{R}=\tau_{R}$,

$$
\left\{\begin{array}{l}
\tau_{m}=\min \left(\tau_{L}, \tau_{1}, \tau_{2}, \tau_{3}, \tau_{R}, \mathcal{T}_{2}\right) \\
\tau_{M}=\max \left(\tau_{L}, \tau_{1}, \tau_{2}, \tau_{3}, \tau_{R}, \mathcal{T}_{2}\right)
\end{array}\right.
$$

the first, third, fifth and last inequalities of (56) then hold true.

It then remains to consider the validity of the second inequality of (56). Let us denote $\mathcal{E}(\theta)$ the entropy dissipation rate across the $\sigma$-wave defined by

$$
\mathcal{E}(\theta)=-\sigma\left(\Sigma\left(\mathbf{v}_{3}^{r}\right)-\Sigma\left(\mathbf{v}_{2}^{r}\right)\right)+\left(\mathcal{F}^{r}\left(\mathbf{v}_{3}^{r}\right)-\mathcal{F}^{r}\left(\mathbf{v}_{2}^{r}\right)\right) .
$$

One has to prove that $\mathcal{E}(\theta) \leq 0$, at least for a relevant choice of $\theta$ which is still a free parameter at this stage. Using the definition of $\Sigma$ and $\mathcal{F}^{r}$, we clearly have

$$
\begin{aligned}
\mathcal{E}(\theta)= & -\sigma\left(\left(e\left(\mathcal{T}_{3}\right)-\frac{p^{2}\left(\mathcal{T}_{3}\right)}{2 a^{2}}\right)-\left(e\left(\mathcal{T}_{2}\right)-\frac{p^{2}\left(\mathcal{T}_{2}\right)}{2 a^{2}}\right)\right) \\
& -\sigma\left(\frac{u_{3}^{2}}{2}-\frac{u_{2}^{2}}{2}\right)+\left(\pi_{3} u_{3}-\pi_{2} u_{2}\right) \\
& -\sigma\left(\frac{\pi_{3}^{2}}{2 a^{2}}-\frac{\pi_{2}^{2}}{2 a^{2}}\right)
\end{aligned}
$$

which also writes

$$
\mathcal{E}(\theta)=-\sigma\left(\left(e\left(\mathcal{T}_{3}\right)-\frac{p^{2}\left(\mathcal{T}_{3}\right)}{2 a^{2}}\right)-\left(e\left(\mathcal{T}_{2}\right)-\frac{p^{2}\left(\mathcal{T}_{2}\right)}{2 a^{2}}\right)\right)-\frac{\sigma^{2}-a^{2}}{2 \sigma a^{2}}\left(\pi_{3}^{2}-\pi_{2}^{2}\right)
$$

since the Rankine-Hugoniot relation $\sigma\left(u_{3}-u_{2}\right)=\pi_{3}-\pi_{2}$ yields

$$
-\sigma\left(\frac{u_{3}^{2}}{2}-\frac{u_{2}^{2}}{2}\right)+\left(\pi_{3} u_{3}-\pi_{2} u_{2}\right)=\frac{\pi_{3}^{2}-\pi_{2}^{2}}{2 \sigma},
$$

in view of the following Leibniz formula

$$
\pi_{3} u_{3}-\pi_{2} u_{2}=\frac{\pi_{3}+\pi_{2}}{2}\left(u_{3}-u_{2}\right)+\frac{u_{3}+u_{2}}{2}\left(\pi_{3}-\pi_{2}\right) .
$$

Using (50) we also have

$$
-\frac{\sigma^{2}-a^{2}}{2 \sigma a^{2}}\left(\pi_{3}^{2}-\pi_{2}^{2}\right)=-\frac{\sigma^{2}-a^{2}}{2 \sigma a^{2}}\left(\pi_{3}-\pi_{2}\right)\left(\pi_{3}+\pi_{2}\right)
$$


with

$$
\left\{\begin{array}{l}
\pi_{3}+\pi_{2}=2 \pi_{*}-a \sigma \theta\left(\tau_{R}-\tau_{L}\right), \\
\pi_{3}-\pi_{2}=-\sigma^{2} \theta\left(\tau_{R}-\tau_{L}\right)
\end{array}\right.
$$

so that we eventually get, recalling that $\mathcal{T}_{3}=\tau_{R}$ :

$$
\begin{aligned}
\mathcal{E}(\theta)= & -\sigma\left(\left(e\left(\tau_{R}\right)-\frac{p^{2}\left(\tau_{R}\right)}{2 a^{2}}\right)-\left(e\left(\mathcal{T}_{2}(\theta)\right)-\frac{p^{2}\left(\mathcal{I}_{2}(\theta)\right)}{2 a^{2}}\right)\right) \\
& +\frac{\sigma^{2}}{2 a\left(a^{2}-\sigma^{2}\right)}\left(\mathcal{I}_{R}-\mathcal{I}_{L}\right)^{2} \theta\left(\theta-\frac{2\left(a^{2}-\sigma^{2}\right)}{a \sigma} \frac{\pi_{*}}{\mathcal{I}_{R}-\mathcal{I}_{L}}\right) .
\end{aligned}
$$

Let us comment on the non linearities in $\theta$ that are involved in the entropy jump condition (59). First observe that the second contribution just resumes to a nice convex quadratic term with respect to $\theta$ (since again $a^{2}>\sigma^{2}>0$ ). Then the remaining non linearity in $\theta$ is obviously related to $\mathcal{T}_{2}(\theta)$. We propose to perform a sharp upper-bound of $\mathcal{E}(\theta)$ that will result in quadratic estimate in $\theta$. By sharp, it is meant that it systematically coincides for the choice $\theta=1$ to the equality (59)

$$
\begin{aligned}
\mathcal{E}(1)= & -\sigma\left(\left(e\left(\tau_{R}\right)-\frac{p^{2}\left(\tau_{R}\right)}{2 a^{2}}\right)-\left(e\left(\tau_{L}\right)-\frac{p^{2}\left(\tau_{L}\right)}{2 a^{2}}\right)\right) \\
& +\frac{\sigma^{2}}{2 a\left(a^{2}-\sigma^{2}\right)}\left(\mathcal{I}_{R}-\mathcal{I}_{L}\right)^{2}\left(1-\frac{2\left(a^{2}-\sigma^{2}\right)}{a \sigma} \frac{\pi_{*}}{\mathcal{I}_{R}-\mathcal{I}_{L}}\right) .
\end{aligned}
$$

This will allow to exactly capture isolated entropy shock solutions. In order to provide such a relevant upper bound for $\mathcal{E}(\theta)$, let us first prove the following lemma.

Lemma 4.1. Under the sub-characteristic condition (57)-(58), the mapping

$$
\mathcal{I} \in\left[\min \left(\mathcal{I}_{2}, \mathcal{I}_{3}\right), \max \left(\mathcal{I}_{2}, \mathcal{I}_{3}\right)\right] \rightarrow f(\mathcal{I}):=\left(e-\frac{p^{2}}{2 a^{2}}\right)(\mathcal{I}(\mathcal{I}))
$$

with $\mathcal{I}_{2}=p\left(\mathcal{T}_{2}\right)+a^{2} \mathcal{T}_{2}$ and $\mathcal{I}_{3}=p\left(\mathcal{T}_{3}\right)+a^{2} \mathcal{T}_{3}$ is well-defined and strictly convex.

Proof. It is first clear that the function $\mathcal{I} \in\left[\min \left(\mathcal{I}_{2}, \mathcal{I}_{3}\right), \max \left(\mathcal{I}_{2}, \mathcal{I}_{3}\right)\right] \rightarrow \mathcal{T}(\mathcal{I})$ is well-defined by the relation

$$
p(\mathcal{T}(\mathcal{I}))+a^{2} \mathcal{T}(\mathcal{I})=\mathcal{I}
$$

under the sub-characteristic condition (57)-(58). We then have

$$
\frac{d}{d \mathcal{I}}\left(e-\frac{p^{2}}{2 a^{2}}\right)(\mathcal{T}(\mathcal{I}))=-\frac{p}{a^{2}}\left(a^{2}+p^{\prime}(\mathcal{T}(\mathcal{I}))\right) \frac{d \mathcal{T}}{d \mathcal{I}}(\mathcal{I})=-\frac{p(\mathcal{T}(\mathcal{I}))}{a^{2}},
$$

and then

$$
\frac{d^{2}}{d \mathcal{I}^{2}}\left(e-\frac{p^{2}}{2 a^{2}}\right)(\mathcal{T}(\mathcal{I}))=-\frac{p^{\prime}(\mathcal{I}(\mathcal{I}))}{a^{2}} \frac{d \mathcal{I}}{d \mathcal{I}}(\mathcal{I})>0
$$

under the sub-characteristic condition (57)-(58) since

$$
\frac{d \mathcal{I}}{d \mathcal{I}}(\mathcal{I})=\frac{1}{a^{2}+p^{\prime}(\mathcal{I}(\mathcal{I}))}
$$


This concludes the proof.

As an immediate consequence, we have

$$
\begin{gathered}
\mathcal{E}(\theta) \leq \\
\theta\left(-\sigma\left(\left(e-\frac{p^{2}}{2 a^{2}}\right)_{R}-\left(e-\frac{p^{2}}{2 a^{2}}\right)_{L}\right)+\frac{\sigma^{2}}{2 a\left(a^{2}-\sigma^{2}\right)}\left(\mathcal{I}_{R}-\mathcal{I}_{L}\right)^{2}\left(\theta-\frac{2\left(a^{2}-\sigma^{2}\right)}{a \sigma} \frac{\pi_{*}}{\mathcal{I}_{R}-\mathcal{I}_{L}}\right)\right) .
\end{gathered}
$$

Indeed, recall that in the case $\sigma>0$ we have $\mathcal{T}_{3}=\mathcal{T}_{R}$ and $\mathcal{T}_{2}(\theta)=\mathcal{T}_{2}\left(\mathcal{I}_{2}(\theta)\right)$ with

$$
\mathcal{I}_{2}(\theta)=(1-\theta) \mathcal{I}_{R}+\theta \mathcal{I}_{L},
$$

so that

$$
f\left(\mathcal{I}_{R}\right)-f\left(\mathcal{I}_{2}(\theta)\right)=f\left(\mathcal{I}_{R}\right)-f\left((1-\theta) \mathcal{I}_{R}+\theta \mathcal{I}_{L}\right) \geq \theta\left(f\left(\mathcal{I}_{R}\right)-f\left(\mathcal{I}_{L}\right)\right)
$$

clearly holds true by convexity of $f$.

We propose the following condensed form for [61),

$$
\mathcal{E}(\theta) \leq \sigma\left(\mathcal{I}_{R}-\mathcal{I}_{L}\right) \theta\left(\frac{\sigma\left(\mathcal{I}_{R}-\mathcal{I}_{L}\right) \theta}{2 a\left(a^{2}-\sigma^{2}\right)}+\mathcal{A}\left(\mathbf{v}_{L}, \mathbf{v}_{R}\right)\right)
$$

with the following clear definition for $\mathcal{A}\left(\mathbf{v}_{L}, \mathbf{v}_{R}\right)$ :

$$
\mathcal{A}\left(\mathbf{v}_{L}, \mathbf{v}_{R}\right)=-\frac{\left(\left(e-\frac{p^{2}}{2 a^{2}}\right)_{R}-\left(e-\frac{p^{2}}{2 a^{2}}\right)_{L}\right)}{\left(\mathcal{I}_{R}-\mathcal{I}_{L}\right)}-\frac{\pi_{*}}{a^{2}} .
$$

Remark 4.2. Observe that the choice $\theta=1$ reduces to the expected equality :

$$
\mathcal{E}(1)=\sigma\left(\mathcal{I}_{R}-\mathcal{I}_{L}\right)\left(\frac{\sigma\left(\mathcal{I}_{R}-\mathcal{I}_{L}\right)}{2 a\left(a^{2}-\sigma^{2}\right)}+\mathcal{A}\left(\mathbf{v}_{L}, \mathbf{v}_{R}\right)\right) .
$$

The proposed upper-bound is thus sharp with respect to our purpose, while just resuming to a factored second order polynomial in $\theta$ in the general situation.

\subsubsection{Definition of $\theta$}

Aim of this short section is to precisely define $\theta \in[0,1]$. From the calculations above, one has to satisfy (62). Let us recall that $\sigma\left(\mathcal{I}_{R}-\mathcal{I}_{L}\right) \geq 0$ by Lemma 3.1 A first natural definition is then given by

$$
\sigma\left(\mathcal{I}_{R}-\mathcal{I}_{L}\right) \theta=\max \left(0, \min \left(\sigma\left(\mathcal{I}_{R}-\mathcal{I}_{L}\right),-2 a\left(a^{2}-\sigma^{2}\right) \mathcal{A}\left(\mathbf{v}_{L}, \mathbf{v}_{R}\right)\right)\right) .
$$

But asking in addition for (53), we are led to propose

$$
\sigma\left(\mathcal{I}_{R}-\mathcal{I}_{L}\right) \theta=\max (0
$$




$$
\left.\min \left(\sigma\left(\mathcal{I}_{R}-\mathcal{I}_{L}\right),-2 a\left(a^{2}-\sigma^{2}\right) \mathcal{A}\left(\mathbf{v}_{L}, \mathbf{v}_{R}\right), \frac{a^{2}|\sigma|(a+|\sigma|)}{a+|\sigma| / 2}(1-\alpha) \tau_{*}\right)\right)
$$

with

$$
\tau_{*}=\left\{\begin{array}{lll}
\tau_{L *} & \text { if } \quad \sigma<0 \\
\tau_{R *} & \text { if } \quad \sigma>0
\end{array}\right.
$$

The precise value of $\alpha \in(0,1)$ will be optimized in the next sections. Note that it is not necessary to define $\theta$ dividing by $\sigma\left(\mathcal{I}_{R}-\mathcal{I}_{L}\right.$ ) (which equals zero when $\left.\tau_{L}=\tau_{R}\right)$ since first, $\theta$ is always multiplied by $\left(\mathcal{I}_{R}-\mathcal{I}_{L}\right)$ in formulas (50) and (51) that define the ARS, and second by $\sigma \neq 0$.

\subsection{Lipschitz-continuity}

This section aims at proving that the proposed ARS is Lipschitz-continuous with respect to the initial states $\mathbf{v}_{L}$ and $\mathbf{v}_{R}$. It is clear from formulas (50) and (51) that the only difficulty may come from the approximate speed of propagation $\sigma$ which is not continuous in the asymptotic regime $\tau_{L} \rightarrow \tau_{R}$ as already stated in section 3.1. However, we are going to prove that the only quantities $\frac{\sigma \theta\left(\mathcal{I}_{R}-\mathcal{I}_{L}\right)}{(a \pm \sigma)}$ and $\frac{\theta\left(\mathcal{I}_{R}-\mathcal{I}_{L}\right)}{\left(a^{2}-\sigma^{2}\right)}=\frac{\sigma \theta\left(\mathcal{I}_{R}-\mathcal{I}_{L}\right)}{\sigma\left(a^{2}-\sigma^{2}\right)}$ involved in (50) and (51) are actually Lipschitz-continuous in this regime.

With this in mind, let us first prove that $\sigma\left(\mathcal{I}_{R}-\mathcal{I}_{L}\right) \theta$ equals $\sigma\left(\mathcal{I}_{R}-\mathcal{I}_{L}\right)$ in the asymptotic regime $\tau_{R} \rightarrow \tau_{L}$. Note first that we clearly have

$$
\begin{gathered}
\lim _{\tau_{R} \rightarrow \tau_{L}}-2 a\left(a^{2}-\sigma^{2}\right) \mathcal{A}\left(\mathbf{v}_{L}, \mathbf{v}_{R}\right)= \\
-2 a\left(a^{2}+p^{\prime}\left(\tau_{L}\right)\right)\left(\frac{\left(e-\frac{p^{2}}{2 a^{2}}\right)^{\prime}\left(\tau_{L}\right)}{\mathcal{I}^{\prime}\left(\tau_{L}\right)}-\frac{p\left(\tau_{L}\right)}{a^{2}}+\frac{1}{2 a}\left(u_{R}-u_{L}\right)\right)
\end{gathered}
$$

that is, since $\mathcal{I}(\tau)=p(\tau)+a^{2} \tau$ and $e^{\prime}(\tau)=-p(\tau)$,

$$
\begin{gathered}
\lim _{\tau_{R} \rightarrow \tau_{L}}-2 a\left(a^{2}-\sigma^{2}\right) \mathcal{A}\left(\mathbf{v}_{L}, \mathbf{v}_{R}\right)= \\
\left(a^{2}+p^{\prime}\left(\tau_{L}\right)\right)\left(4 p\left(\tau_{L}\right)-\left(u_{R}-u_{L}\right)\right) .
\end{gathered}
$$

On the other hand,

$$
\begin{gathered}
\lim _{\tau_{R} \rightarrow \tau_{L}} \frac{a^{2}|\sigma|(a+|\sigma|)}{a+|\sigma| / 2}(1-\alpha) \tau_{*}= \\
\frac{a^{2} \sqrt{-p^{\prime}\left(\tau_{L}\right)}\left(a+\sqrt{-p^{\prime}\left(\tau_{L}\right)}\right)}{a+\sqrt{-p^{\prime}\left(\tau_{L}\right)} / 2}(1-\alpha) \tau_{*}
\end{gathered}
$$

with

$$
\tau_{*}=\left\{\begin{array}{ccc}
\tau_{L}+\frac{u_{R}-u_{L}}{2 a} & \text { if } & \sigma<0, \\
\tau_{L}+\frac{u_{R}-u_{L}}{2 a} & \text { if } & \sigma>0 .
\end{array}\right.
$$


Let us then observe that by definition of $\sigma$ and $\mathcal{I}$,

$$
\sigma\left(\mathcal{I}_{R}-\mathcal{I}_{L}\right)= \pm \sqrt{-\frac{p_{R}-p_{L}}{\tau_{R}-\tau_{L}}}\left(p_{R}-p_{L}+a^{2}\left(\tau_{R}-\tau_{L}\right)\right)
$$

which clearly gives

$$
\lim _{\tau_{R} \rightarrow \tau_{L}} \sigma\left(\mathcal{I}_{R}-\mathcal{I}_{L}\right)=0 .
$$

As an immediate consequence, $\sigma\left(\mathcal{I}_{R}-\mathcal{I}_{L}\right) \theta=\sigma\left(\mathcal{I}_{R}-\mathcal{I}_{L}\right)=0$ in the asymptotic regime $\tau_{R} \rightarrow \tau_{L}$ by definition (65).

It is now easy to prove that in the limit $\tau_{R} \rightarrow \tau_{L}$,

$$
\frac{\sigma \theta\left(\mathcal{I}_{R}-\mathcal{I}_{L}\right)}{(a \pm \sigma)}=\frac{\sigma\left(\mathcal{I}_{R}-\mathcal{I}_{L}\right)}{(a \pm \sigma)}
$$

is Lipschitz-continuous, since

$$
\frac{\sigma}{(a \pm \sigma)} \frac{\left(\mathcal{I}_{R}-\mathcal{I}_{L}\right)}{\tau_{R}-\tau_{L}}
$$

is clearly bounded. Indeed,

$$
\lim _{\tau_{R} \rightarrow \tau_{L}} \frac{\sigma}{(a \pm \sigma)} \frac{\left(\mathcal{I}_{R}-\mathcal{I}_{L}\right)}{\tau_{R}-\tau_{L}}=\frac{\epsilon \sqrt{-p^{\prime}\left(\tau_{L}\right)}\left(a^{2}+p^{\prime}\left(\tau_{L}\right)\right)}{a \pm \epsilon \sqrt{-p^{\prime}\left(\tau_{L}\right)}}
$$

with $\epsilon= \pm 1$.

In the same way, we have in the limit $\tau_{R} \rightarrow \tau_{L}$

$$
\frac{\theta\left(\mathcal{I}_{R}-\mathcal{I}_{L}\right)}{a^{2}-\sigma^{2}}=\frac{\sigma \theta\left(\mathcal{I}_{R}-\mathcal{I}_{L}\right)}{\sigma\left(a^{2}-\sigma^{2}\right)}=\frac{\sigma\left(\mathcal{I}_{R}-\mathcal{I}_{L}\right)}{\sigma\left(a^{2}-\sigma^{2}\right)}=\frac{\left(\mathcal{I}_{R}-\mathcal{I}_{L}\right)}{\left(a^{2}-\sigma^{2}\right)},
$$

which is Lipschitz-continuous since

$$
\frac{1}{\left(a^{2}-\sigma^{2}\right)} \frac{\left(\mathcal{I}_{R}-\mathcal{I}_{L}\right)}{\tau_{R}-\tau_{L}}
$$

is clearly bounded. Indeed

$$
\lim _{\tau_{R} \rightarrow \tau_{L}} \frac{1}{\left(a^{2}-\sigma^{2}\right)} \frac{\left(\mathcal{I}_{R}-\mathcal{I}_{L}\right)}{\tau_{R}-\tau_{L}}=\frac{1}{\left(a^{2}+p^{\prime}\left(\tau_{L}\right)\right)}\left(a^{2}+p^{\prime}\left(\tau_{L}\right)\right)=1 .
$$

\subsection{The case of an isolated entropy shock solution}

Let us assume that the initial states $\mathbf{v}_{L}$ and $\mathbf{v}_{R}$ are such that they can be joined by an admissible entropy shock solution propagating with velocity $s$, assuming $s>0$ without restriction from Galilean symmetry. This means that we have the validity of the following Rankine-Hugoniot relations

$$
\left\{\begin{array}{l}
-s\left(\tau_{R}-\tau_{L}\right)-\left(u_{R}-u_{L}\right)=0 \\
-s\left(u_{R}-u_{L}\right)+\left(p_{R}-p_{L}\right)=0
\end{array}\right.
$$


together with the validity of the following entropy inequality

$$
-s\left(\mathcal{U}_{R}-\mathcal{U}_{L}\right)+\left(\mathcal{F}_{R}-\mathcal{F}_{L}\right) \leq 0,
$$

or equivalently by well-known consideration :

$$
-s\left(\left(e_{R}-e_{L}\right)-\frac{p_{L}+p_{R}}{2}\left(\tau_{R}-\tau_{L}\right)\right) \leq 0 .
$$

Note that in this case $\sigma$ defined by (39) exactly coincides with the exact speed of propagation $s$ and satisfies in particular

$$
s^{2}=\sigma^{2}=-\frac{p_{R}-p_{L}}{\tau_{R}-\tau_{L}} .
$$

Our purpose is to prove that

$$
\mathbf{v}_{1}=\mathbf{v}_{2}=\mathbf{v}_{L} \quad \text { and } \quad \mathbf{v}_{3}=\mathbf{v}_{R}
$$

This will be done into two steps. We will first prove that such initial states lead by definition (65) to the choice

$$
\sigma\left(\mathcal{I}_{R}-\mathcal{I}_{L}\right) \theta=\sigma\left(\mathcal{I}_{R}-\mathcal{I}_{L}\right),
$$

or equivalently $\theta=1$, and second that this choice gives (70). Let us first state some basic relations easily obtained from (66) and (67).

Lemma 4.3. Let us assume that (66) and (67) hold true. Then we have

$$
\left\{\begin{array}{l}
\sigma\left(\mathcal{I}_{R}-\mathcal{I}_{L}\right)=\sigma\left(a^{2}-\sigma^{2}\right)\left(\tau_{R}-\tau_{L}\right), \\
\left(a^{2}-\sigma^{2}\right)\left(\tau_{R}-\tau_{L}\right)=a^{2}\left(\tau_{R *}-\tau_{L *}\right), \\
u_{*}=\frac{1}{2 a}\left((a-\sigma) u_{R}+(a+\sigma) u_{L}\right), \\
\pi_{*}=\frac{1}{2}\left(p_{L}+p_{R}\right)+\frac{\sigma a}{2}\left(\tau_{R}-\tau_{L}\right) .
\end{array}\right.
$$

Let us now prove that $\sigma\left(\mathcal{I}_{R}-\mathcal{I}_{L}\right) \theta=\sigma\left(\mathcal{I}_{R}-\mathcal{I}_{L}\right)$.

Lemma 4.4. Let us assume that [66) and (67) hold true. Assume that $\alpha$ is such that

$$
\left\{\begin{array}{c}
\alpha \leq \frac{\tau_{R *}}{\tau_{L *}} \quad \text { if } \quad \sigma<0, \\
\alpha \leq \frac{\tau_{L *}}{\tau_{R *}} \quad \text { if } \quad \sigma>0 .
\end{array}\right.
$$

Then, by definition (65) we have

$$
\sigma\left(\mathcal{I}_{R}-\mathcal{I}_{L}\right) \theta=\sigma\left(\mathcal{I}_{R}-\mathcal{I}_{L}\right) .
$$

Proof. Let us first prove that

$$
\sigma\left(\mathcal{I}_{R}-\mathcal{I}_{L}\right) \leq-2 a\left(a^{2}-\sigma^{2}\right) \mathcal{A}\left(\mathbf{v}_{L}, \mathbf{v}_{R}\right),
$$


that is, since again $\mathcal{I}_{R}-\mathcal{I}_{L}=\left(a^{2}-\sigma^{2}\right)\left(\tau_{r}-\tau_{L}\right)$

$$
\sigma\left(\tau_{R}-\tau_{L}\right) \leq-2 a \mathcal{A}\left(\mathbf{v}_{L}, \mathbf{v}_{R}\right) .
$$

By definition of $\mathcal{A}\left(\mathbf{v}_{L}, \mathbf{v}_{R}\right)$ and $\pi_{*}$, this is equivalent to

$$
\sigma\left(\tau_{R}-\tau_{L}\right) \leq \frac{2 a\left(\left(e-\frac{p^{2}}{2 a^{2}}\right)_{R}-\left(e-\frac{p^{2}}{2 a^{2}}\right)_{L}\right)}{\left(a^{2}-\sigma^{2}\right)\left(\tau_{R}-\tau_{L}\right)}+\frac{p_{L}+p_{R}}{a}-\left(u_{R}-u_{L}\right),
$$

and by the first equation in (66) (recall that $s=\sigma$ ) to

$$
0 \leq \frac{2 a\left(\left(e-\frac{p^{2}}{2 a^{2}}\right)_{R}-\left(e-\frac{p^{2}}{2 a^{2}}\right)_{L}\right)}{\left(a^{2}-\sigma^{2}\right)\left(\tau_{R}-\tau_{L}\right)}+\frac{p_{L}+p_{R}}{a} .
$$

Recalling that shock solutions with $\sigma>0$ comes with the property $\tau_{R}>\tau_{L}$, we multiply the above inequality by $\sigma\left(a^{2}-\sigma^{2}\right)\left(\tau_{R}-\tau_{L}\right)>0$ and use (69) to infer the equivalent form

$$
-\sigma\left(\left(e_{R}-e_{L}\right)-\frac{p_{L}+p_{R}}{2}\left(\tau_{R}-\tau_{L}\right)\right) \leq 0,
$$

which is nothing but (68).

It remains to prove that

$$
\sigma\left(\mathcal{I}_{R}-\mathcal{I}_{L}\right)=\sigma\left(a^{2}-\sigma^{2}\right)\left(\tau_{R}-\tau_{L}\right) \leq \frac{a^{2}|\sigma|(a+|\sigma|)}{a+|\sigma| / 2}(1-\alpha) \tau_{*},
$$

with

$$
\tau_{*}=\left\{\begin{array}{lll}
\tau_{L *} & \text { if } & \sigma<0 \\
\tau_{R *} & \text { if } & \sigma>0,
\end{array}\right.
$$

that is to say

$$
\sigma(a-|\sigma|)(a+|\sigma|)\left(\tau_{R}-\tau_{L}\right) \leq \frac{a^{2}|\sigma|(a+|\sigma|)}{a+|\sigma| / 2}(1-\alpha) \tau_{*},
$$

or again

$$
\sigma(a-|\sigma|)\left(\tau_{R}-\tau_{L}\right) \leq \frac{a^{2}|\sigma|}{a+|\sigma| / 2}(1-\alpha) \tau_{*} .
$$

A sufficient condition is

$$
\sigma(a-|\sigma|)(a+|\sigma|)\left(\tau_{R}-\tau_{L}\right) \leq a^{2}|\sigma|(1-\alpha) \tau_{*} .
$$

that is

$$
\sigma\left(a^{2}-\sigma^{2}\right)\left(\tau_{R}-\tau_{L}\right) \leq a^{2}|\sigma|(1-\alpha) \tau_{*},
$$

or by Lemma 4.3

$$
\sigma a^{2}\left(\tau_{R *}-\tau_{L *}\right) \leq a^{2}|\sigma|(1-\alpha) \tau_{*},
$$

that is

$$
\sigma\left(\tau_{R *}-\tau_{L *}\right) \leq|\sigma|(1-\alpha) \tau_{*} .
$$


If $\sigma>0$ (which gives $\tau_{R}>\tau_{L}$ and by Lemma $4.3 \tau_{R *}>\tau_{L *}$ ), we get

$$
\alpha \leq \frac{\tau_{L *}}{\tau_{R *}}
$$

while if $\sigma<0$ (which gives $\tau_{R}<\tau_{L}$ and by Lemma $4.3 \tau_{R *}<\tau_{L *}$ ), we get

$$
\alpha \leq \frac{\tau_{R *}}{\tau_{L *}},
$$

which concludes the proof.

At last and to conclude this section, let us show that $\mathbf{v}_{1}=\mathbf{v}_{2}=\mathbf{v}_{L}$ and $\mathbf{v}_{3}=\mathbf{v}_{R}$. It just amounts to replace $\sigma\left(\mathcal{I}_{R}-\mathcal{I}_{L}\right) \theta$ by

$$
\sigma\left(\mathcal{I}_{R}-\mathcal{I}_{L}\right)=\left(a^{2}-\sigma^{2}\right)\left(\tau_{R}-\tau_{L}\right)
$$

as proposed in (501). Let us consider for instance the case $\sigma>0$ without restriction so that $\mathbf{v}_{-}=\mathbf{v}_{2}$ and $\mathbf{v}_{+}=\mathbf{v}_{3}$. Using the definition of $\pi_{*}$ established in Lemma 4.3 we easily get

$$
\pi_{-}=\pi_{2}=\pi_{1}=p_{L} \quad \text { and } \quad \pi_{+}=\pi_{3}=p_{R} .
$$

Using next the definition of $u_{*}$ established in Lemma 4.3 we easily get

$$
u_{-}=u_{2}=u_{1}=p_{L} \quad \text { and } \quad u_{+}=u_{3}=u_{R} .
$$

As an immediate consequence we have by (50)

$$
\tau_{1}=\tau_{L} \quad \text { and } \quad \tau_{3}=\tau_{R} .
$$

At last and still by (50) we have (recall that $\sigma>0$ for the sake of specificity)

$$
\tau_{2}=\tau_{3}-\left(\tau_{R}-\tau_{L}\right)=\tau_{R}-\left(\tau_{R}-\tau_{L}\right)=\tau_{L}
$$

\subsection{Summary}

The next proposition sums up the properties satisfied by the proposed ARS based on a generalized Suliciu relaxation approach.

Proposition 4.5. Under the sub-characteristic condition [57), the proposed approximate Riemann solver

(i) is exact for isolated admissible shock waves,

(ii) is entropy satisfying,

(iii) is Lipschitz-continuous with respect to the initial states $\mathbf{v}_{\mathbf{L}}$ and $\mathbf{v}_{\mathbf{R}}$,

(iv) is $L^{1}$ stable in the sense that the intermediate states belong to the phase space. 
About the choice of $\alpha$ and the sub-characteristic condition (57). Assume that $\alpha$ is chosen as follows :

$$
\left\{\begin{array}{c}
\alpha=\frac{\tau_{R *}}{\tau_{L *}} \quad \text { if } \quad \sigma<0, \\
\alpha=\frac{\tau_{L *}}{\tau_{R *}} \quad \text { if } \quad \sigma>0 .
\end{array}\right.
$$

Then, we have by Lemma 3.1

$$
\begin{cases}\tau_{1} \geq \tau_{L *}, & \text { for all } \sigma, \\ \tau_{3} \geq \tau_{R *}, & \text { for all } \sigma, \\ \tau_{2} \geq \tau_{R *}, & \text { for all } \sigma<0 \\ \tau_{2} \geq \tau_{L *}, & \text { for all } \sigma>0,\end{cases}
$$

so that the sub-characteristic condition (57) simply writes

$$
a^{2}>\max _{\tau \in\left\lfloor\tau_{L}, \tau_{L *}, \tau_{R *}, \tau_{R}\right\rceil}-p^{\prime}(\tau) .
$$

This is nothing but the usual sub-characteristic condition (32) of the classical relaxation approach (without the additional $\sigma$-wave).

\section{$5 \quad$ Numerical schemes}

In this section, we propose two different numerical schemes using the approximate Riemann solver derived in the previous sections. The first one is a classical approximate Godunov-type method as described in subsection 2.2 with the proposed approximate Riemann solver, and the second one is an original Glimmtype scheme with random sampling strategy, the objective of which is to give sharp shock discontinuities (without numerical diffusion). We first briefly describe this method and show numerical results for both methods.

Let us first recall that the classical Godunov-type method defines the update value of the unknown at the new time step by simply averaging at time $t=\Delta t$ the solution made of the juxtaposition of the approximate Riemann solutions defined as proposed in the previous sections and set at each cell interfaces. With clear notations, we more precisely have

$$
\mathbf{u}_{j}^{n+1}=\frac{1}{\Delta x} \int_{x_{j-1 / 2}}^{x_{j+1 / 2}} \tilde{\mathbf{u}}(x, \Delta t) d t .
$$

This scheme is then conservative and entropy-satisfying from the previous sections.

In the Glimm-type method, we first propose to average at time $t=\Delta t$ the approximate solution made of the juxtaposition of the proposed Riemann solutions on the modified cells $\left[\bar{x}_{j-1 / 2}, \bar{x}_{j+1 / 2}\right]$ defined by

$$
\bar{x}_{j+1 / 2}=x_{j+1 / 2}+\sigma_{j+1 / 2} \Delta t \text {. }
$$


More precisely, we propose to set

$$
\overline{\mathbf{u}}_{j}^{n+1}=\frac{1}{\overline{\Delta x}_{j}} \int_{\bar{x}_{j-1 / 2}}^{\bar{x}_{j+1 / 2}} \tilde{\mathbf{u}}(x, \Delta t) d t
$$

with

$$
\overline{\Delta x}_{j}=\bar{x}_{j+1 / 2}-\bar{x}_{j-1 / 2}
$$

as illustrated on the figure below. This way, no spurious intermediate state (and then no numerical diffusion) is created when dealing with isolated admissible shocks since the approximate Riemann solver is exact in this case. In order to recover the initial mesh and define $\mathbf{u}_{j}^{n+1}$ without introducing numerical diffusion, we then proceed with a random sampling procedure similar to the random choice method [11] (see also for similar strategies [5], [8], 9] and the references therein). More precisely, let be given $\left(a_{n}\right)_{n}$ a well-distributed random sequence within $(0, \Delta x)(e . g$. the van der Corput sequence [11]), we simply set

$$
\mathbf{u}_{j}^{n+1}=\left\{\begin{array}{lll}
\overline{\mathbf{u}}_{j-1}^{n+1} & \text { if } & a_{n+1} \in\left(0, \frac{\Delta t}{\Delta x} \sigma_{j-1 / 2}^{+}\right) \\
\overline{\mathbf{u}}_{j}^{n+1} & \text { if } & a_{n+1} \in\left[\frac{\Delta t}{\Delta x} \sigma_{j-1 / 2}^{+}, 1+\frac{\Delta t}{\Delta x} \sigma_{j+1 / 2}^{-}\right) \\
\overline{\mathbf{u}}_{j+1}^{n+1} & \text { if } & a_{n+1} \in\left[1+\frac{\Delta t}{\Delta x} \sigma_{j+1 / 2}^{-}, 1\right)
\end{array}\right.
$$

where $\sigma_{j+1 / 2}$ is the speed of propagation of the additional wave coming from $x_{j+1 / 2}$ while $\sigma_{j+1 / 2}^{+}=\max \left(\sigma_{j+1 / 2}, 0\right)$ and $\sigma_{j+1 / 2}^{-}=\min \left(\sigma_{j+1 / 2}, 0\right)$.

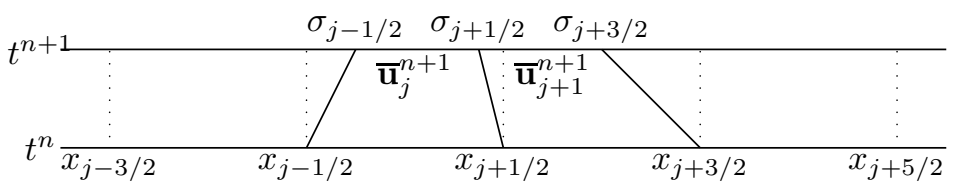

We now propose three numerical illustrations associated with Riemann initial data with left state $\mathbf{v}_{L}=\left(\tau_{L}, u_{L}\right)$ and right state $\mathbf{v}_{R}=\left(\tau_{R}, u_{R}\right)$. The computational domain is $(0,1)$ and the initial discontinuity is located at point $x=0$. The pressure law is $p(\tau)=\tau^{\gamma}$ with $\gamma=2$ and the mesh is made of 100 points. The first test case corresponds to an isolated shock discontinuity with $\mathbf{v}_{L}=(1,0)$ and $\mathbf{v}_{R}=(2,-\sqrt{3} / 2)$. The second test case corresponds to a Riemann solution with a rarefaction wave and a shock discontinuity, and is associated with the choice $\mathbf{v}_{L}=(0.3,0)$ and $\mathbf{v}_{R}=(0.6,0)$. At last, the third test case has two shock discontinuities, one propagating with a negative velocity and one with a positive velocity, and corresponds to $\mathbf{v}_{L}=(0.5,2)$ and $\mathbf{v}_{R}=(0.6,0)$. The results are given on Figures 6 [ 7 and 8 for both methods. As expected, we observe that the Godunov-type method provides good numerical results with usual numerical diffusion, while the Glimm-type method gives sharp discontinuities without numerical diffusion. 

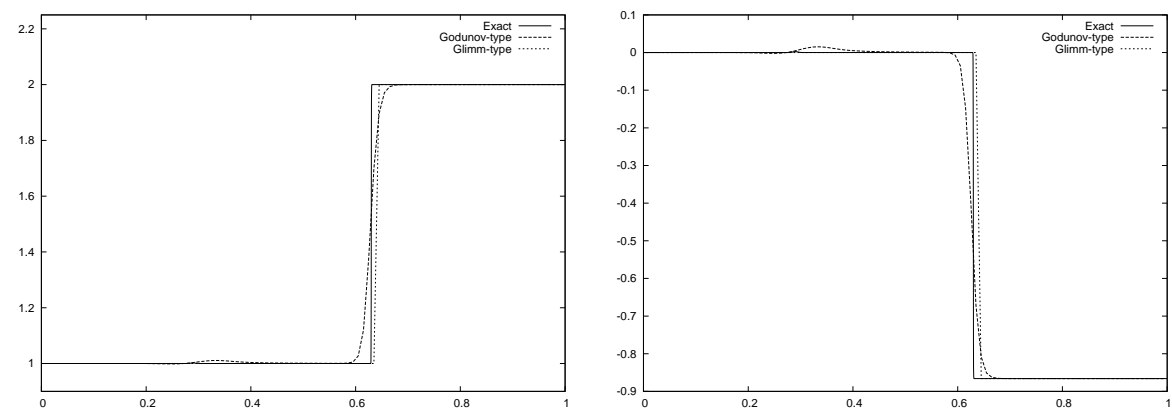

Figure 6: Test 1: $\tau$ (left) and $u$ (right) at time $t=0.15$.
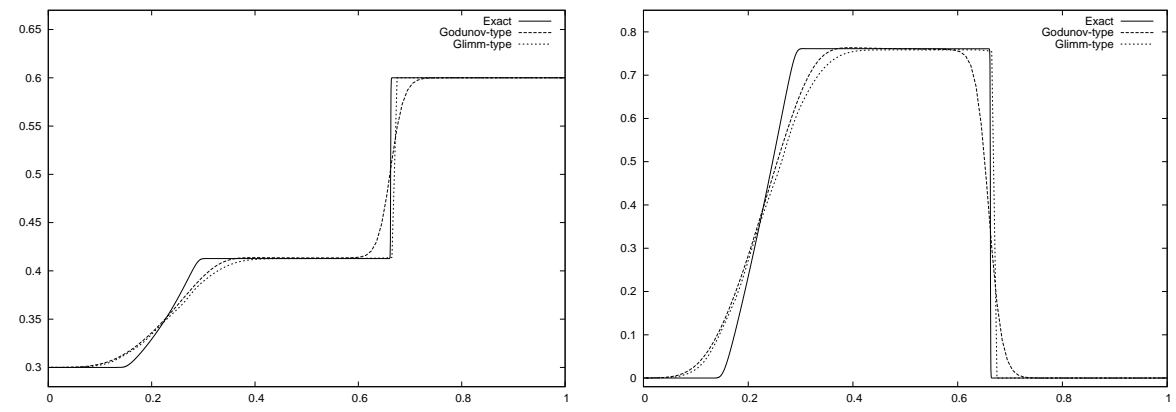

Figure 7: Test 2: $\tau$ (left) and $u$ (right) at time $t=0.04$.
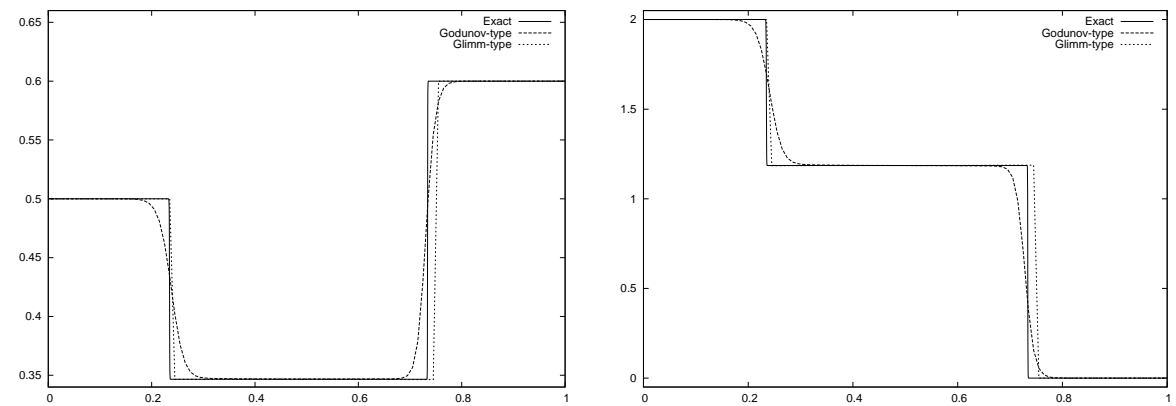

Figure 8: Test $3: \tau$ (left) and $u$ (right) at time $t=0.05$. 


\section{References}

[1] F. Bouchut, A reduced stability condition for nonlinear relaxation to conservation laws, J. Hyp. Diff. Eq., vol 1(1), pp 149-170, (2004)

[2] F. Bouchut, T. Morales, Semi-discrete entropy satisfying approximate Riemann solvers. The case of the Suliciu relaxation approximation, Journal of Scientific Computing, 41(3):483-509, (2009)

[3] A. Bressan, Hyperbolic Systems of Conservation Laws: The Onedimensional Cauchy Problem, Oxford University Press, (2000)

[4] C. Chalons and F. Coquel, Navier-Stokes equations with several independent pressure laws and explicit predictor-corrector schemes, Numerisch Math., vol 101(3), pp 451-478 (2005)

[5] Chalons C. and Coquel F., Capturing infinitely sharp discrete shock profiles with the Godunov scheme, Proceedings of the Eleventh International Conference on Hyperbolic Problems. Theory, Numerics, Applications, S. Benzoni-Gavage and D. Serre (Eds), Springer, pp 363-370 (2008)

[6] C. Chalons, F. Coquel, E. Godlewski, P-A Raviart, N. Seguin. Godunovtype schemes for hyperbolic systems with parameter dependent source. The case of Euler system with friction. Math. Models Methods Appl. Sci., (2010)

[7] C. Chalons and J.-F. Coulombel. Relaxation approximation of the Euler equations. Journal of Mathematical Analysis and Applications, 348(2), pp. 872-893, (2008)

[8] C. Chalons and P. Goatin, Godunov scheme and sampling technique for computing phase transitions in traffic flow modeling, Interfaces and Free Boundaries, vol 10, no. 2, pp 197-221 (2008)

[9] C. Chalons and P. Goatin, Transport-Equilibrium schemes for computing contact discontinuities in traffic flow modeling, Commun. Math. Sci., No 3 , pp 533-551 (2007)

[10] G.-Q. Chen, C.-D. Levermore and T.-P. Liu, Hyperbolic conservation laws with stiff relaxation terms and entropy, Comm. Pure Appl. Math, vol 48(7), pp 787-830 (1995)

[11] P. Collela, Glimm's method for gas dynamics, SIAM J. Sci. Stat. Comput., vol 3, pp 76-110 (1982)

[12] F. Coquel, E. Godlewski, A. In, B. Perthame and P. Rascle, Some new Godunov and relaxation methods for two phase flows, Proceedings of an International conference on Godunov methods : Theory and Applications, Kluwer Academic/Plenum Publishers (2001) 
[13] A. Harten, P.-D. Lax, B. van Leer: On upstream Differencing and Godunovtype Schemes for Hyperbolic Conservation Laws. SIAM Review, 25(1): 35$61(1983)$

[14] E. Godlewsky, P.-A. Raviart, Numerical approximation of hyperbolic systems of conservation laws. Springer (1995)

[15] S. Jin and Z. Xin, The Relaxation Schemes for Systems of Conservation Laws in Arbitrary Space Dimension, Comm. Pure. Appl. Math., vol 48, pp 235-276 (1995)

[16] P.-G LeFloch, Hyperbolic systems of conservation laws: the theory of classical and nonclassical shock waves. Birkhäuser (2002)

[17] R. Natalini, Convergence to equilibrium for the relaxation approximations of conservation laws. Comm. Pure Appl. Math. 49, pp. 1-30, (1996)

[18] P.-L. Roe, Approximate Riemann Solvers, Parameter Vectors, and Difference Schemes. J. Comput. Phys., 43:357-372, (1981)

[19] I. Suliciu, On the thermodynamics of fluids with relaxation and phase transitions. Fluids with relaxation. Int. J. Engrg. Sci. 36, pp. 921-947, (1998)

[20] E. F. Toro, Riemann solvers and numerical methods for fluid dynamics: A practical introduction. Springer-Verlag, Berlin, (1997) 